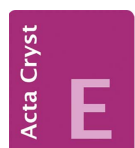

CRYSTALLOGRAPHIC COMMUNICATIONS

ISSN 2056-9890

Received 1 February 2016

Accepted 10 February 2016

Edited by J. Simpson, University of Otago, New Zealand

Keywords: crystal structure; Schiff base; bisimine; thiophene.

CCDC references: 1452795; 1452794; $1452793 ; 1452792$

Supporting information: this article has supporting information at journals.iucr.org/e

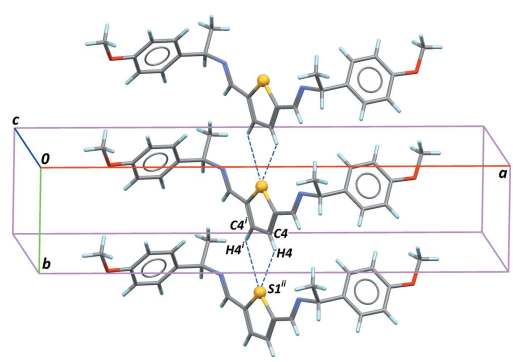

OPEN $\odot$ ACCESS

\section{Crystal structures of four chiral imine-substituted thiophene derivatives}

\author{
Guadalupe Hernández-Téllez, ${ }^{\mathrm{a}}$ Sylvain Bernès, ${ }^{\mathrm{b} *}$ Angel Mendoza, ${ }^{\mathrm{c}}$ Francisco Javier \\ Ríos-Merino, ${ }^{\mathrm{c}}$ Gloria E. Moreno, ${ }^{\mathrm{a}}$ Oscar Portillo ${ }^{\mathrm{a}}$ and René Gutiérrez ${ }^{\mathrm{a}}$
}

\begin{abstract}
a'Laboratorio de Síntesis de Complejos, Facultad de Ciencias Químicas, Universidad Autónoma de Puebla, A.P. 1067, 72001 Puebla, Pue., Mexico, 'bnstituto de Física, Universidad Autónoma de Puebla, Av. San Claudio y 18 Sur, 72570 Puebla, Pue., Mexico, and ${ }^{\mathrm{c} C e n t r o ~ d e ~ Q u i ́ m i c a, ~ I n s t i t u t o ~ d e ~ C i e n c i a s, ~ U n i v e r s i d a d ~ A u t o ́ n o m a ~ d e ~ P u e b l a, ~} 72570$ Puebla, Pue., Mexico. ${ }^{*}$ Correspondence e-mail: sylvain_bernes@hotmail.com
\end{abstract}

A series of thiophenes substituted in positions 2 and 5 by imine groups have been synthesized using a solvent-free approach, and their crystal structures determined. The substituents are chiral groups, and the expected absolute configuration for each molecule was confirmed by refinement of the Flack parameter. The compounds are 2,5-bis $[(S)-(+)-(1,2,3,4$-tetrahydronaphthalen-1yl)imino]thiophene, $\mathrm{C}_{26} \mathrm{H}_{26} \mathrm{~N}_{2} \mathrm{~S}$, (I), 2,5-bis $\{[(R)-(-)$-1-(4-methoxyphenyl)ethyl]iminomethyl\}thiophene, $\mathrm{C}_{24} \mathrm{H}_{26} \mathrm{~N}_{2} \mathrm{O}_{2} \mathrm{~S}$, (II), 2,5-bis $\{[(R)-(-)$-1-(4-fluorophenyl)ethyl $]$ iminomethyl $\}$ thiophene, $\mathrm{C}_{22} \mathrm{H}_{20} \mathrm{~F}_{2} \mathrm{~N}_{2} \mathrm{~S}$, (III), and 2,5-bis $\{[(S)-(+)-1-(4-$ chlorophenyl)ethyl]iminomethyl\}thiophene, $\mathrm{C}_{22} \mathrm{H}_{20} \mathrm{Cl}_{2} \mathrm{~N}_{2} \mathrm{~S}$, (IV). A common feature of all four molecules is the presence of twofold symmetry. For (I), which crystallizes in the triclinic space group $P 1$, this symmetry is non-crystallographic, but for (II) in $C 2$ and the isomorphous structures (III) and (IV) that crystallize in $P 2{ }_{1} 2_{1} 2$, the twofold symmetry is crystallographically imposed with one half of each molecule in the asymmetric unit. The comparable molecular symmetry in the four structures is also reflected in similar packing, with molecules aggregated to form chains through weak $\mathrm{C}-\mathrm{H} \cdots \mathrm{S}$ interactions.

\section{Chemical context}

Thiophenedicarbaldehydes have a variety of applications (Dean, 1982a,b), for instance in the synthesis of annulenones and polyenyl-substituted thiophenes (Sargent \& Cresp, 1975), in the preparation of macrocyclic ligands for bimetallic complexes that are able to mimic enzymes (Nelson et al., 1983), in crown ether chemistry (Cram \& Trueblood, 1981) and, more recently, in the preparation of azomethines for photovoltaic applications (Bolduc et al., 2013a,b; Petrus et al., 2014). In regard to this latter application, most of the conjugated materials used in organic electronics are synthesized using time-consuming Suzuki-, Wittig-, or Heck-type coupling reactions that require expensive catalysts, stringent reaction conditions, and tedious purification processes. In order to afford a more economic route towards organic photovoltaic materials, Schiff bases derived from 2,5-thiophenedicarbaldehyde as the conjugated linker unit have recently been used. The azomethine bond, which is isoelectronic with the vinyl bond and possesses similar optoelectronic and thermal properties, is easily accessible through the Schiff condensation under near ambient reaction conditions (Morgan et al., 1987; Pérez Guarìn et al., 2007; Sicard et al., 2013). 


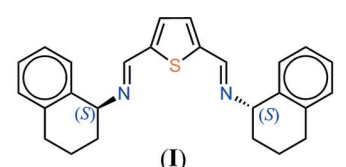

(I)<smiles>C[C@H](N=Cc1ccc(C=N[C@@H](C)c2ccc(F)cc2)s1)c1ccc(F)cc1</smiles><smiles>CC(N=Cc1ccc(C=N[C@@H](C)c2ccc(Cl)cc2)s1)c1ccc(Cl)cc1</smiles>

We report here the synthesis and X-ray characterization of such thiophene derivatives, as a continuation of a partially published record (Bernès et al., 2013; Mendoza et al., 2014). We are improving a general solvent-free approach for these syntheses, recognising that ecological aspects in organic chemistry have become a priority, in order to minimize the quantity of toxic waste and by-products, and to decrease the amount of solvent in the reaction media or during work-up (Tanaka \& Toda, 2000; Noyori, 2005).

In the synthesis of the thiophenes reported here, the Schiff condensation generates a single by-product, water, and a onestep recrystallization affords the pure substituted thiophene in nearly quantitative yields. Our protocol may be readily extended to any low molecular weight 2,5-susbtituted thiophene, providing that a liquid amine is used for the condensation. In the present work, the starting material is 2,5thiophenedicarbaldehyde, a low melting-point compound (m.p. $=388-390 \mathrm{~K}$ ), and four chiral amines were used. We took advantage of the anomalous dispersion of the sulfur sites to confirm that the configuration of the chiral amine is retained during the condensation.

\section{Structural commentary}

The first compound was synthesized using $(S)$-(+)-1-aminotetraline. The Schiff base (I), $\mathrm{C}_{26} \mathrm{H}_{26} \mathrm{~N}_{2} \mathrm{~S}$, crystallizes in the space group $P 1$, with the expected absolute configuration

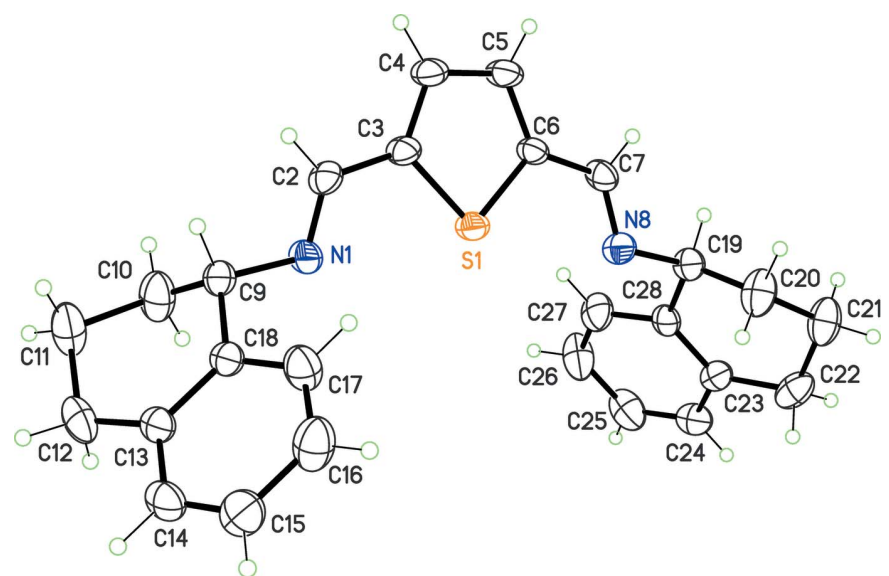

Figure 1

The molecular structure of (I), with displacement ellipsoids for non-H atoms at the $30 \%$ probability level.

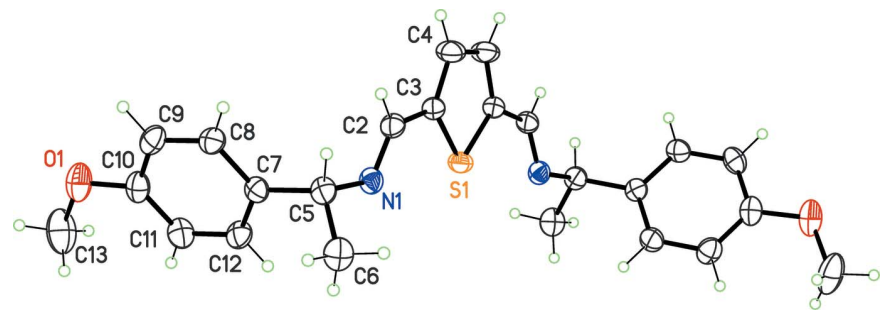

Figure 2

The molecular structure of (II), with displacement ellipsoids for non- $\mathrm{H}$ atoms at the $30 \%$ probability level. Non-labeled atoms are generated by symmetry code $(1-x, y, 1-z)$.

(Fig. 1). The general shape of the molecule displays a pseudotwofold axis, passing through the $\mathrm{S}$ atom and the midpoint of the thiophene $\mathrm{C}-\mathrm{C} \sigma$-bond. As a consequence, the independent benzene rings are placed above and below the thiophene ring, and are inclined to one another at a dihedral angle of $73.76(15)^{\circ}$. The central core containing the thiophene ring and the imine bonds is virtually planar, and the imine bonds are substituted by the tetralin ring systems, which present the same conformation. The aliphatic rings C9-C13/C18 and C19$\mathrm{C} 23 / \mathrm{C} 28$ each have a half-chair conformation.

Compound (II), $\mathrm{C}_{24} \mathrm{H}_{26} \mathrm{~N}_{2} \mathrm{O}_{2} \mathrm{~S}$, was obtained using $(R)-(+)$ (4-methoxy)phenylethylamine as the chiral component in the Schiff condensation. The twofold molecular axis, which was a latent symmetry in the case of (I), is a true crystallographic symmetry in (II), and this compound crystallizes in the space group C2 (Fig. 2). The asymmetric unit thus contains half a

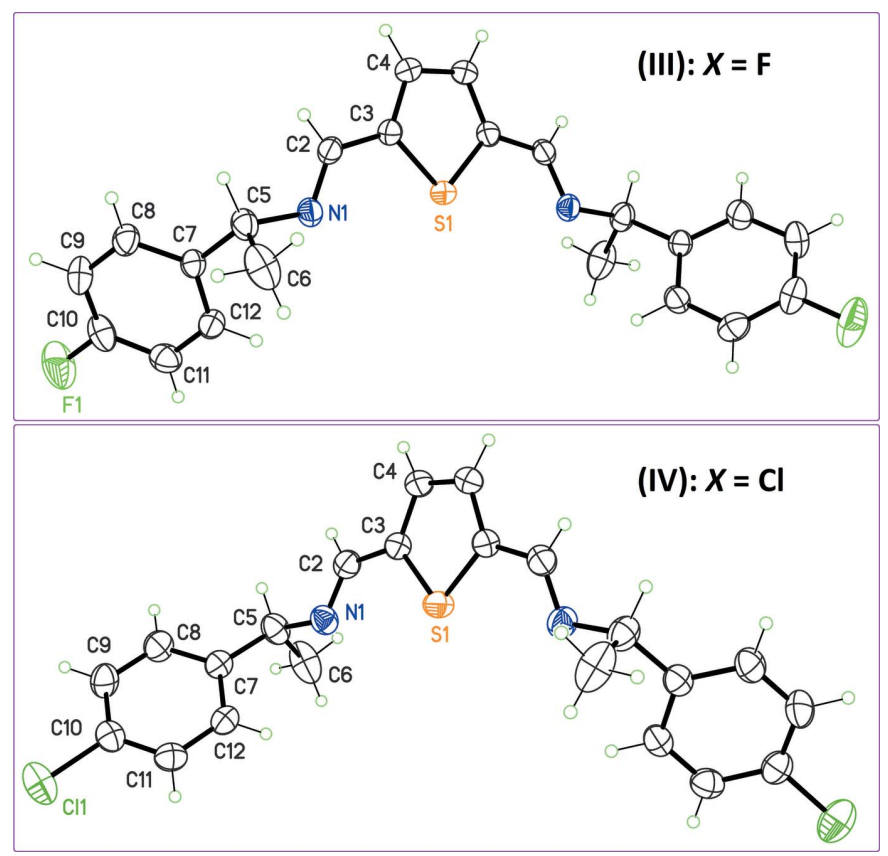

Figure 3

The molecular structures of isomorphous compounds (III) and (IV), with displacement ellipsoids for non- $\mathrm{H}$ atoms at the $30 \%$ probability level. Notice the different configuration for chiral center C5 in (III) and (IV). Non-labeled atoms are generated by symmetry codes $(1-x,-y, z)$ and $(1-x, 2-y, z)$ for (III) and (IV), respectively. 
Table 1

Comparison of $\mathrm{C}-\mathrm{H} \cdots \mathrm{S}$ hydrogen bonds $\left(\AA{ }^{\circ}\right)$ in compounds (I)-(IV).

\begin{tabular}{llllll}
\hline Compound & Contact & $\mathrm{C}-\mathrm{H}$ & $\mathrm{H} \cdots \mathrm{S}$ & $\mathrm{C} \cdots \mathrm{S}$ & $\mathrm{C}-\mathrm{H} \cdots \mathrm{S}$ \\
\hline (I) & $\mathrm{C} 4-\mathrm{H} 4 A \cdots \mathrm{S} 1^{\mathrm{i}}$ & 0.93 & 3.00 & $3.562(5)$ & 121 \\
(I) & $\mathrm{C} 5-\mathrm{H} 5 A \cdots \mathrm{S} 1^{\mathrm{i}}$ & 0.93 & 2.97 & $3.547(5)$ & 122 \\
& & & & \\
(II) & $\mathrm{C} 4-\mathrm{H} 4 A \cdots \mathrm{S} 1^{\text {ii }}$ & 0.93 & 2.99 & $3.572(3)$ & 122 \\
(III) & $\mathrm{C} 4-\mathrm{H} 4 A \cdots \mathrm{S} 1^{\text {iii }}$ & 0.93 & 3.15 & $3.743(3)$ & 124 \\
(IV) & $\mathrm{C} 4-\mathrm{H} 4 A \cdots \mathrm{S} 1^{\text {iv }}$ & 0.93 & 3.23 & $3.828(4)$ & 124 \\
\hline
\end{tabular}

Symmetry codes: (i) $x+1, y, z$; (ii) $x, y+1, z$; (iii) $x, y, z+1$; (iv) $x, y, z-1$.

molecule, and the molecular conformation for the complete molecule is similar to that of (I). The benzene rings have a free relative orientation, since these rings are not fused in a bicyclic system, as in (I); the dihedral angle between symmetry-related rings is $61.30(7)^{\circ}$.

Compounds (III) and (IV), synthesized with enantiomerically pure (4-halogen)phenylethylamines (halogen $=\mathrm{F}, \mathrm{Cl}$ ) are isomorphous and crystallize with orthorhombic unit cells. The latent twofold symmetry of (I) is again observed, since both molecules lie on the crystallographic twofold axes of the space group $P 2{ }_{1}{ }_{1} 2$ (Fig. 3). The dihedral angle between the benzene rings is close to that observed for (II): 64.18 (8) ${ }^{\circ}$ for (III) and $62.03(9)^{\circ}$ for (IV). The same Schiff base but with $\mathrm{Br}$ as the halogen substituent has been published previously (Mendoza et al., 2014), but is not isomorphous with (III) and (IV). Instead, this molecule was found to crystallize in the space group $C 2$, with unit-cell parameters and a crystal structure very similar to those of (II). A systematic trend is thus emerging for these 2,5-substituted thiophenes, related to the potential twofold molecular symmetry: they have a strong tendency to crystallize in space groups that include at least one $C_{2}$ axis, such as $C 2$ and $P 2{ }_{1} 2{ }_{1} 2$ for the chiral crystals. This trend extends to achiral molecules, which also have twofold crystallographic symmetry in the space group C2/c (Kudyakova et al., 2011; Suganya et al., 2014; Boyle et al., 2015; Moussallem et al., 2015). The features shared by these related compounds could also be a signature of a propensity towards polymorphism between monoclinic and orthorhombic systems.

The difference between non-crystallographic symmetry in (I) and exact $C_{2}$ molecular symmetry in (II)-(IV) is also reflected in the degree of conjugation between thiophene rings and imine bonds. For (I), dihedral angles between the thiophene and $\mathrm{C}=\mathrm{N}-\mathrm{C}^{*}$ mean planes $\left(\mathrm{C}^{*}\right.$ is the chiral $\mathrm{C}$ atom bonded to the imine functionality) are $6.9(7)$ and $1.9(6)^{\circ}$. Other crystals have a symmetry restriction, inducing a small deconjugation of the imine bonds. The corresponding dihedral angles with the thiophene rings are $8.5(4), 10.1(3)$, and $9.8(3)^{\circ}$, for (II), (III) and (IV), respectively.

\section{Supramolecular features}

Although all compounds have benzene rings, neither $\pi-\pi$ nor $\mathrm{C}-\mathrm{H} \cdots \pi$ contacts stabilize the crystal structures. However, these compounds share a common supramolecular feature. Lone pairs of $\mathrm{S}$ atoms interact with thiophenic $\mathrm{CH}$ groups of a

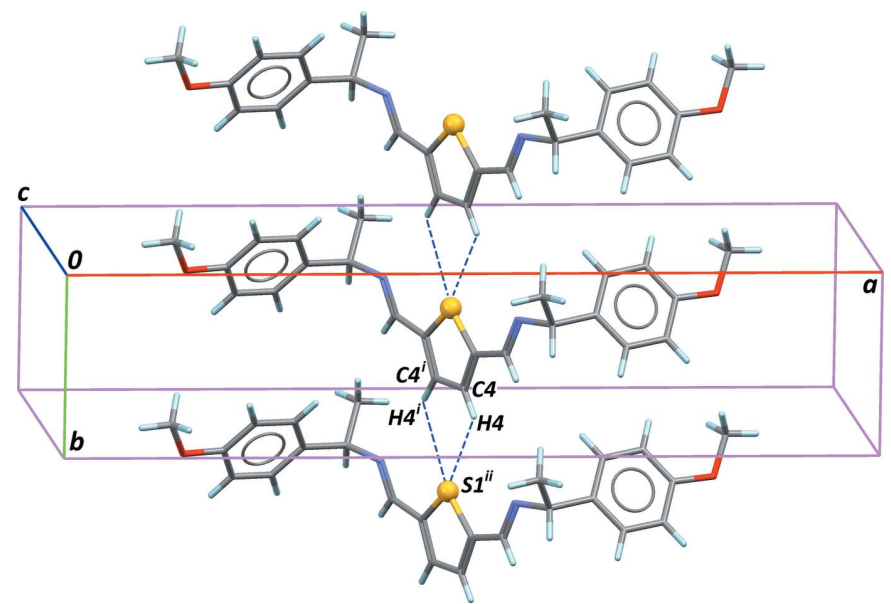

Figure 4

Part of the crystal structure of (II), showing $\mathrm{C}-\mathrm{H} \cdots \mathrm{S}$ hydrogen bonds (dashed lines) linking molecules along [010]. [Symmetry codes: (i) $1-x$, $y, 1-z$; (ii) $x, 1+y, z$.]

neighboring molecule in the crystal, forming chains along the short cell axes: [100] for (I), [010] for (II) and [001] for (III) and (IV). An example is presented in Fig. 4, for compound (II). These bifurcated S. . C $-\mathrm{H}$ contacts have a significant strength for (I), perhaps as a consequence of the relaxed molecular symmetry in space group $P 1$. The contacts are weaker for (II), (III) and (IV), which have a geometry restrained by the crystallographic symmetry (Table 1 ).

\section{Database survey}

Many thiophenes substituted in the 2 and 5 positions by imine groups have been characterized; however, almost all were achiral compounds. X-ray structures have been reported mostly in space group C2/c (Suganya et al., 2014; Kudyakova et al., 2011, 2012; Bolduc et al., 2013b). Other represented space groups for achiral molecules are $P 2_{1}$ (Skene \& Dufresne, 2006) and $P 2_{1} / c$ (Wiedermann et al., 2005). Finally, a single case of a molecule presenting mirror symmetry has been described (Fridman \& Kaftory, 2007), in space group Pnma.

The group of chiral molecules belonging to this family is much less populated, with two examples reported by our group in this journal. Both are molecules with the $C_{2}$ point group and crystallize in space groups $C 2$ (Mendoza et al., 2014) and $P 22_{1} 2_{1}$ (Bernès et al., 2013).

\section{Synthesis and crystallization}

Synthesis. The chiral amines used for the Schiff condensation were obtained directly from suppliers: $(S)-(+)-1,2,3,4$-tetrahydro-1-naphthylamine for $(\mathrm{I}),(R)-(+)-1-(4-m e t h o x y p h e n-$ yl)ethylamine for (II), (R)-(+)-1-(4-fluorophenyl)ethylamine for (III) and (S)-(-)-1-(4-chlorophenyl)ethylamine for (IV). 2,5-Thiophenedicarbaldehyde (100 $\mathrm{mg}, 0.71 \mathrm{mmol})$ and the chiral amine $(1.4 \mathrm{mmol})$ in a 1:2 molar ratio were mixed at room temperature under solvent-free conditions, giving lightyellow (II and IV), colorless (III) or light-brown (IV) solids, in 
Table 2

Experimental details.

(I)

Crystal data

Chemical formula

$M_{\mathrm{r}}$

Crystal system, space group

Temperature $(\mathrm{K})$

$a, b, c(\AA)$

$\alpha, \beta, \gamma\left(^{\circ}\right)$

$V\left(\AA^{3}\right)$

Z

Radiation type

$\mu\left(\mathrm{mm}^{-1}\right)$

Crystal size (mm)

Data collection

Diffractometer

Absorption correction

$T_{\min }, T_{\max }$

No. of measured, independent and observed $[I>2 \sigma(I)]$ reflections

$R_{\text {int }}$

$(\sin \theta / \lambda)_{\max }\left(\AA^{-1}\right)$

Refinement

$R\left[F^{2}>2 \sigma\left(F^{2}\right)\right], w R\left(F^{2}\right), S$

No. of reflections

No. of parameters

No. of restraints

$\mathrm{H}$-atom treatment

$\Delta \rho_{\max }, \Delta \rho_{\min }\left(\mathrm{e} \AA^{-3}\right)$

Absolute structure

Absolute structure parameter
(II)

$\mathrm{C}_{24} \mathrm{H}_{26} \mathrm{~N}_{2} \mathrm{O}_{2} \mathrm{~S}$
406.53
Monoclinic, $C 2$
298
$25.3917(13), 5.9488(3)$,
$\quad 7.5623(4)$
$90,97.174(4), 90$
$1133.34(10)$
2
Mo $K \alpha$
0.16
$0.45 \times 0.33 \times 0.12$

Agilent Xcalibur (Atlas, Gemini)

Agilent Xcalibur (Atlas, Gemini)

Analytical CrysAlis PRO,

(Agilent, 2013)

$0.969,0.992$

6689, 4036, 2958

\subsection{0}

0.618

$0.058,0.127,1.02$
4036
262
3
H-atom parameters
$\quad$ constrained
$0.31,-0.19$
Flack $x$ determined using 962
$\quad$ quotients
$\quad\left[\left(I^{+}\right)-\left(I^{-}\right)\right] /\left[\left(I^{+}\right)+\left(I^{-}\right)\right]$
$\quad($ Parsons et al., 2013$)$
$-0.12(7)$

Agilent, 2013)

$0.973,0.993$

6341, 2221, 1892

\subsection{7}

0.618

$0.036,0.085,1.02$
2221
134
1
H-atom parameters
$\quad$ constrained
$0.11,-0.17$
Flack $x$ determined using 708
$\quad$ quotients
$\quad\left[\left(I^{+}\right)-\left(I^{-}\right)\right] /\left[\left(I^{+}\right)+\left(I^{-}\right)\right]$
$\quad($ Parsons et al., 2013$)$
$-0.02(4)$

Analytical (CrysAlis PRO;
(III)

$\mathrm{C}_{22} \mathrm{H}_{20} \mathrm{~F}_{2} \mathrm{~N}_{2} \mathrm{~S}$
382.46
Orthorhombic, $P 2{ }_{1} 2{ }_{1} 2$
298
$21.1153(16), 7.7846(6)$
$\quad \quad 6.1343(5)$
$90,90,90$

$1008.32(14)$
2
$\mathrm{Mo} \mathrm{K \alpha}$
0.19
$0.89 \times 0.47 \times 0.33$

(IV)

$\mathrm{C}_{22} \mathrm{H}_{20} \mathrm{Cl}_{2} \mathrm{~N}_{2} \mathrm{~S}$
415.36
Orthorhombic, $P 2{ }_{1} 2{ }_{1} 2$
298
$21.893(2), 7.9212(6)$,
$\quad \quad 6.2315(4)$
$90,90,90$
$1080.66(15)$
2
Mo $K \alpha$
0.41
$0.52 \times 0.40 \times 0.07$

Agilent Xcalibur (Atlas, Gemini)

Analytical CrysAlis PRO, (Agilent, 2013) $0.904,0.958$

12336, 2067, 1591

Agilent Xcalibur (Atlas,
Gemini)
Multi-scan CrysAlis PRO,
$\quad$ (Agilent, 2013)
$0.692,1.000$
$14195,2743,1534$
0.058
0.692

$\begin{array}{ll}0.044,0.092,1.06 & 0.052,0.117,1.01 \\ 2067 & 2743 \\ 124 & 124 \\ 0 & 0 \\ \text { H-atom parameters } & \text { H-atom parameters } \\ \quad \text { constrained } & \text { constrained } \\ 0.15,-0.25 & 0.13,-0.17 \\ \text { Flack } x \text { determined using } 518 & \text { Flack } x \text { determined using } 465 \\ \quad \text { quotients } & \text { quotients } \\ \quad\left[\left(I^{+}\right)-\left(I^{-}\right)\right] /\left[\left(I^{+}\right)+\left(I^{-}\right)\right] & {\left[\left(I^{+}\right)-\left(I^{-}\right)\right] /\left[\left(I^{+}\right)+\left(I^{-}\right)\right]} \\ \quad(\text { Parsons } \text { et al., } 2013) & (\text { Parsons } \text { et al., } 2013) \\ 0.07(6) & 0.10(6)\end{array}$

Computer programs: CrysAlis PRO (Agilent, 2013), SHELXS97 (Sheldrick, 2008), SHELXT (Sheldrick, 2015a), SHELXL2014 (Sheldrick, 2015b) and Mercury (Macrae et al., 2008).

95-97\% yields. The crude solids were recrystallized from $\mathrm{CH}_{2} \mathrm{Cl}_{2}$, affording colorless crystals of (I)-(IV).

Spectroscopy. (I): m.p. $437-438$ K. $[\alpha]_{\mathrm{D}}^{20}=+655.4(c=1$, $\left.\mathrm{CHCl}_{3}\right)$. FTIR: $1616 \mathrm{~cm}^{-1}(\mathrm{C}=\mathrm{N}) .{ }^{1} \mathrm{H}$ NMR $(500 \mathrm{MHz}$, $\left.\mathrm{CHCl}_{3} / \mathrm{TMS}\right): \delta=1.76-1.86(m, 2 \mathrm{H} ; H$-al $), 1.96-2.06(m, 6 \mathrm{H}$; $H$-al), 2.74-2.90 ( $m, 4 \mathrm{H} ; H$-al), 4.51 ( $t, 2 \mathrm{H} ; H$-al $), 6.98-7.02(m$, 2H; $H$-ar), 7.09-7.15 ( $m, 6 \mathrm{H} ; H$-ar), $7.28(s, 2 \mathrm{H} ; H$-ar $), 8.36(s$, $2 \mathrm{H} ; H \mathrm{C}=\mathrm{N}) .{ }^{13} \mathrm{C}$ NMR: $\delta=19.7,29.3,31.1,67.7(C$-al $), 125.7$, 126.9, 128.7, 129.1, 129.6, 136.8, 137.1, 145.1 (C-ar), 153.1 $(\mathrm{H} C=\mathrm{N})$. MS-EI: $m / z=398\left(M^{+}\right)$.

(II): m.p. $405-406 \mathrm{~K} .[\alpha]_{\mathrm{D}}^{20}=-626.8\left(c=1, \mathrm{CHCl}_{3}\right)$. FTIR: $1631 \mathrm{~cm}^{-1}(\mathrm{C}=\mathrm{N}) .{ }^{1} \mathrm{H}$ NMR $\left(500 \mathrm{MHz}, \mathrm{CHCl}_{3} / \mathrm{TMS}\right): \delta=1.53$ $\left(d, 6 \mathrm{H} ; \mathrm{CHCH}_{3}\right), 3.78\left(s, 6 \mathrm{H} ; \mathrm{OCH}_{3}\right), 4.47\left(q, 2 \mathrm{H} ; \mathrm{CHCH}_{3}\right)$, 6.85-6.88 ( $m, 4 \mathrm{H} ; H$-ar), 7.19 ( $s, 2 \mathrm{H} ; H$-ar), 7.29-7.32 ( $m, 4 \mathrm{H}$; $H$-ar $), 8.33(s, 2 \mathrm{H} ; H \mathrm{C}=\mathrm{N}) .{ }^{13} \mathrm{C} \mathrm{NMR}: \delta=24.8\left(\mathrm{CHCH}_{3}\right), 55.2$ $\left(\mathrm{OCH}_{3}\right), 68.1\left(\mathrm{CHCH}_{3}\right), 113.7,127.6,129.6,137.1,145.2,152.1$ $(C$-ar $), 158.5(\mathrm{HC}=\mathrm{N})$. MS-EI: $m / z=406\left(M^{+}\right)$.

(III): m.p. $420-421 \mathrm{~K} \cdot[\alpha]_{\mathrm{D}}^{20}=-542.5\left(c=1, \mathrm{CHCl}_{3}\right)$. FTIR: $1621 \mathrm{~cm}^{-1}(\mathrm{C}=\mathrm{N}) .{ }^{1} \mathrm{H}$ NMR $\left(500 \mathrm{MHz}, \mathrm{CHCl}_{3} / \mathrm{TMS}\right): \delta=1.53$ $\left(d, 6 \mathrm{H} ; \mathrm{CHCH}_{3}\right), 4.49$ ( $\left.q, 2 \mathrm{H} ; \mathrm{CHCH}_{3}\right), 7.00-7.38(m, 10 \mathrm{H}$;
$H$-ar $), 8.37(s, 2 \mathrm{H} ; \mathrm{HC}=\mathrm{N}) .{ }^{13} \mathrm{C} \mathrm{NMR:} \delta=25.2\left(\mathrm{CHCH}_{3}\right), 68.7$ $\left(\mathrm{CHCH}_{3}\right), 115.2\left(d, J_{\mathrm{F}-\mathrm{C}}=21.2 \mathrm{~Hz} ; C\right.$-ar $), 128.1\left(d, J_{\mathrm{F}-\mathrm{C}}=\right.$ $8.7 \mathrm{~Hz} ; C$-ar $), 130.1$ ( $C$-ar $), 140.7\left(d, J_{\mathrm{F}-\mathrm{C}}=2.5 \mathrm{~Hz} ; C\right.$-ar $), 145.1$ $(C$-ar $), 161.1\left(d, J_{\mathrm{F}-\mathrm{C}}=242.5 \mathrm{~Hz} ; C\right.$-ar $), 152.5(\mathrm{HC}=\mathrm{N})$. MS-EI: $m / z=382\left(M^{+}\right)$.

(IV): m.p. $434-435$ K. $[\alpha]_{\mathrm{D}}^{20}=+726.5\left(c=1, \mathrm{CHCl}_{3}\right)$. FTIR: $1623 \mathrm{~cm}^{-1}(\mathrm{C}=\mathrm{N}) .{ }^{1} \mathrm{H}$ NMR (500 MHz, $\left.\mathrm{CHCl}_{3} / \mathrm{TMS}\right): \delta=1.53$ $\left(d, 6 \mathrm{H} ; \mathrm{CHCH}_{3}\right), 4.48\left(q, 2 \mathrm{H} ; \mathrm{CHCH}_{3}\right), 7.23-7.35(m, 10 \mathrm{H} ; \mathrm{H}-$ ar), $8.37(s, 2 \mathrm{H} ; H \mathrm{C}=\mathrm{N}) .{ }^{13} \mathrm{C}$ NMR: $\delta=25.2\left(\mathrm{CHCH}_{3}\right), 68.7$ $\left(\mathrm{CHCH}_{3}\right), 128.0,128.6,130.2,132.5,143.5,145.1$ (C-ar), 152.7 $(\mathrm{HC}=\mathrm{N})$.

\section{Refinement}

Crystal data, data collection and structure refinement details are summarized in Table 2. No unusual issues appeared, and refinements were carried out on non-restricted models. All $\mathrm{H}$ atoms were placed in calculated positions, and refined as riding on their carrier $\mathrm{C}$ atoms, with $\mathrm{C}-\mathrm{H}$ bond lengths fixed to 0.93 (aromatic $\mathrm{CH}$ ), 0.96 (methyl $\mathrm{CH}_{3}$ ), 0.97 (methylene 
$\mathrm{CH}_{2}$ ), or $0.98 \AA$ (methine $\mathrm{CH}$ ). Isotropic displacement parameters were calculated as $U_{\text {iso }}(\mathrm{H})=1.5 U_{\text {eq }}(\mathrm{C})$ for methyl $\mathrm{H}$ atoms and $U_{\text {iso }}(\mathrm{H})=1.2 U_{\text {eq }}(\mathrm{C})$ for other $\mathrm{H}$ atoms. For all compounds, the absolute configuration was based on the refinement of the Flack parameter (Parsons et al., 2013), confirming that the configuration of the chiral amine used as the starting material was retained during the Schiff condensation.

\section{Acknowledgements}

Support from VIEP-UAP is acknowledged.

\section{References}

Agilent (2013). CrysAlis PRO. Agilent Technologies Inc., Santa Clara, CA, USA.

Bernès, S., Hernández-Téllez, G., Sharma, M., Portillo-Moreno, O. \& Gutiérrez, R. (2013). Acta Cryst. E69, o1428.

Bolduc, A., Al Ouahabi, A., Mallet, C. \& Skene, W. G. (2013a). J. Org. Chem. 78, 9258-9269.

Bolduc, A., Dufresne, S. \& Skene, W. G. (2013b). Acta Cryst. C69, 1196-1199.

Boyle, R., Crundwell, G. \& Glagovich, N. M. (2015). Acta Cryst. E71, o403.

Cram, D. J. \& Trueblood, K. N. (1981). Top. Curr. Chem. 98, 43106.

Dean, F. M. (1982a). Adv. Heterocycl. Chem. 30, 167-238.

Dean, F. M. (1982b). Adv. Heterocycl. Chem. 31, 237-344.

Fridman, N. \& Kaftory, M. (2007). Pol. J. Chem. 81, 825-832.

Kudyakova, Yu. S., Burgart, Ya. V. \& Saloutin, V. I. (2011). Chem. Heterocycl. Compd. 47, 558-563.
Kudyakova, Y. S., Burgart, Y. V., Slepukhin, P. A. \& Saloutin, V. I. (2012). Mendeleev Commun. 22, 284-286.

Macrae, C. F., Bruno, I. J., Chisholm, J. A., Edgington, P. R., McCabe, P., Pidcock, E., Rodriguez-Monge, L., Taylor, R., van de Streek, J. \& Wood, P. A. (2008). J. Appl. Cryst. 41, 466-470.

Mendoza, A., Bernès, S., Hernández-Téllez, G., Portillo-Moreno, O. \& Gutiérrez, R. (2014). Acta Cryst. E70, o345.

Morgan, P. W., Kwolek, S. L. \& Pletcher, T. C. (1987). Macromolecules, 20, 729-739.

Moussallem, C., Allain, M., Mallet, C., Gohier, F. \& Frère, P. (2015). J. Fluor. Chem. 178, 34-39.

Nelson, S. M., Esho, F., Lavery, A. \& Drew, M. G. B. (1983). J. Am. Chem. Soc. 105, 5693-5695.

Noyori, R. (2005). Chem. Commun. pp. 1807-1811.

Parsons, S., Flack, H. D. \& Wagner, T. (2013). Acta Cryst. B69, 249259.

Pérez Guarìn, S. A., Bourgeaux, M., Dufresne, S. \& Skene, W. G. (2007). J. Org. Chem. 72, 2631-2643.

Petrus, M. L., Bouwer, R. K. M., Lafont, U., Athanasopoulos, S., Greenham, N. C. \& Dingemans, T. J. (2014). J. Mater. Chem. A, 2, 9474-9477.

Sargent, M. V. \& Cresp, T. M. (1975). Fortschritte Chem. Forschung, 57, 111-143.

Sheldrick, G. M. (2008). Acta Cryst. A64, 112-122.

Sheldrick, G. M. (2015a). Acta Cryst. A71, 3-8.

Sheldrick, G. M. (2015b). Acta Cryst. C71, 3-8.

Sicard, L., Navarathne, D., Skalski, T. \& Skene, W. G. (2013). $A d v$. Funct. Mater. 23, 3549-3559.

Skene, W. G. \& Dufresne, S. (2006). Acta Cryst. E62, o1116-o1117.

Suganya, S., Velmathi, S. \& MubarakAli, D. (2014). Dyes Pigments, 104, 116-122.

Tanaka, K. \& Toda, F. (2000). Chem. Rev. 100, 1025-1074.

Wiedermann, J., Kirchner, K. \& Mereiter, K. (2005). Private communication (refcode NAWMAA). CCDC, Cambridge, England. 


\section{supporting information}

Acta Cryst. (2016). E72, 350-354 [doi:10.1107/S2056989016002516]

\section{Crystal structures of four chiral imine-substituted thiophene derivatives}

Guadalupe Hernández-Téllez, Sylvain Bernès, Angel Mendoza, Francisco Javier Ríos-Merino, Gloria E. Moreno, Oscar Portillo and René Gutiérrez

Computing details

For all compounds, data collection: CrysAlis PRO (Agilent, 2013); cell refinement: CrysAlis PRO (Agilent, 2013); data reduction: CrysAlis PRO (Agilent, 2013). Program(s) used to solve structure: SHELXS97 (Sheldrick, 2008) for (I); SHELXT (Sheldrick, 2015a) for (II), (III), (IV). For all compounds, program(s) used to refine structure: SHELXL2014 (Sheldrick, 2015b); molecular graphics: Mercury (Macrae et al., 2008); software used to prepare material for publication: SHELXL2014 (Sheldrick, 2015b).

(I) 2,5-Bis $[(S)-(+)-(1,2,3,4-t e t r a h y d r o-1-n a p h t h y l)$ imino]thiophene

Crystal data

$\mathrm{C}_{26} \mathrm{H}_{26} \mathrm{~N}_{2} \mathrm{~S}$

$M_{r}=398.55$

Triclinic, $P 1$

$a=5.9093(4) \AA$

$b=7.6258(5) \AA$

$c=12.6570(8) \AA$

$\alpha=87.802(5)^{\circ}$

$\beta=78.329(5)^{\circ}$

$\gamma=87.427(5)^{\circ}$

$V=557.76(6) \AA^{3}$

$Z=1$

\section{Data collection}

Agilent Xcalibur (Atlas, Gemini) diffractometer

Radiation source: Enhance (Mo) X-ray Source Graphite monochromator

Detector resolution: 10.5564 pixels $\mathrm{mm}^{-1}$ $\omega$ scans

Absorption correction: analytical

CrysAlis PRO, (Agilent, 2013)

$T_{\min }=0.969, T_{\max }=0.992$

Refinement

Refinement on $F^{2}$

Least-squares matrix: full

$R\left[F^{2}>2 \sigma\left(F^{2}\right)\right]=0.058$

$w R\left(F^{2}\right)=0.127$

$S=1.02$

4036 reflections
$F(000)=212$

$D_{\mathrm{x}}=1.187 \mathrm{Mg} \mathrm{m}^{-3}$

Melting point: $437 \mathrm{~K}$

Mo $K \alpha$ radiation, $\lambda=0.71073 \AA$

Cell parameters from 2148 reflections

$\theta=3.3-22.6^{\circ}$

$\mu=0.16 \mathrm{~mm}^{-1}$

$T=298 \mathrm{~K}$

Plate, colorless

$0.34 \times 0.12 \times 0.06 \mathrm{~mm}$

6689 measured reflections

4036 independent reflections

2958 reflections with $I>2 \sigma(I)$

$R_{\text {int }}=0.040$

$\theta_{\max }=26.1^{\circ}, \theta_{\min }=3.1^{\circ}$

$h=-7 \rightarrow 7$

$k=-9 \rightarrow 9$

$l=-15 \rightarrow 15$

262 parameters

3 restraints

0 constraints

Primary atom site location: structure-invariant direct methods 
Secondary atom site location: difference Fourier map

Hydrogen site location: inferred from neighbouring sites

$\mathrm{H}$-atom parameters constrained $w=1 /\left[\sigma^{2}\left(F_{\mathrm{o}}^{2}\right)+(0.0525 P)^{2}\right]$

where $P=\left(F_{\mathrm{o}}^{2}+2 F_{\mathrm{c}}^{2}\right) / 3$
$(\Delta / \sigma)_{\max }<0.001$
$\Delta \rho_{\max }=0.31 \mathrm{e} \AA^{-3}$
$\Delta \rho_{\min }=-0.19 \mathrm{e} \AA^{-3}$

Absolute structure: Flack $x$ determined using 962 quotients $\left[\left(I^{+}\right)-\left(I^{-}\right)\right] /\left[\left(I^{+}\right)+\left(I^{-}\right)\right]$(Parsons et al., 2013)

Absolute structure parameter: -0.12 (7)

Fractional atomic coordinates and isotropic or equivalent isotropic displacement parameters $\left(\AA^{2}\right)$

\begin{tabular}{|c|c|c|c|c|}
\hline & $x$ & $y$ & $z$ & $U_{\text {iso }} * / U_{\text {eq }}$ \\
\hline $\mathrm{S} 1$ & $0.66581(19)$ & $0.49640(17)$ & $0.11819(12)$ & 0.0488 (4) \\
\hline N1 & $0.5097(7)$ & $0.7980(6)$ & 0.2625 & 0.0507 (12) \\
\hline $\mathrm{C} 2$ & $0.7239(10)$ & $0.7657(7)$ & $0.2474(4)$ & 0.0490 (13) \\
\hline $\mathrm{H} 2 \mathrm{~A}$ & 0.8132 & 0.8300 & 0.2834 & $0.059 *$ \\
\hline $\mathrm{C} 3$ & $0.8355(8)$ & $0.6297(7)$ & 0.1747 (4) & 0.0469 (13) \\
\hline $\mathrm{C} 4$ & $1.0622(9)$ & $0.5892(7)$ & $0.1402(5)$ & $0.0575(15)$ \\
\hline $\mathrm{H} 4 \mathrm{~A}$ & 1.1792 & 0.6461 & 0.1628 & $0.069^{*}$ \\
\hline $\mathrm{C} 5$ & $1.1042(8)$ & $0.4510(7)$ & $0.0658(5)$ & $0.0595(15)$ \\
\hline $\mathrm{H} 5 \mathrm{~A}$ & 1.2513 & 0.4075 & 0.0348 & $0.071 *$ \\
\hline C6 & $0.9068(8)$ & $0.3894(6)$ & 0.0450 & $0.0425(12)$ \\
\hline $\mathrm{C} 7$ & $0.8786(9)$ & $0.2528(7)$ & $-0.0268(4)$ & $0.0503(14)$ \\
\hline H7A & 1.0094 & 0.1943 & -0.0651 & $0.060 *$ \\
\hline N8 & $0.6816(8)$ & $0.2106(6)$ & -0.0390 & $0.0518(12)$ \\
\hline C9 & $0.4190(9)$ & $0.9453(7)$ & $0.3325(4)$ & $0.0518(13)$ \\
\hline H9A & 0.5365 & 0.9745 & 0.3730 & $0.062 *$ \\
\hline $\mathrm{C} 10$ & $0.3728(12)$ & $1.1032(8)$ & $0.2631(5)$ & $0.0772(18)$ \\
\hline H10A & 0.2751 & 1.0713 & 0.2143 & $0.093 *$ \\
\hline H10B & 0.5174 & 1.1431 & 0.2201 & $0.093 *$ \\
\hline $\mathrm{C} 11$ & $0.2537(13)$ & $1.2501(8)$ & 0.3345 & 0.0802 (19) \\
\hline H11A & 0.3449 & 1.2749 & 0.3875 & $0.096 *$ \\
\hline H11B & 0.2407 & 1.3560 & 0.2909 & $0.096^{*}$ \\
\hline C12 & $0.0161(11)$ & $1.1958(8)$ & $0.3911(5)$ & $0.0682(18)$ \\
\hline $\mathrm{H} 12 \mathrm{~A}$ & -0.0468 & 1.2796 & 0.4462 & $0.082 *$ \\
\hline H12B & -0.0847 & 1.1991 & 0.3393 & $0.082 *$ \\
\hline C13 & $0.0174(9)$ & $1.0143(7)$ & $0.4429(4)$ & $0.0486(14)$ \\
\hline $\mathrm{C} 14$ & $-0.1721(10)$ & $0.9610(9)$ & $0.5196(5)$ & $0.0620(16)$ \\
\hline H14A & -0.2950 & 1.0410 & 0.5406 & $0.074 *$ \\
\hline C15 & $-0.1846(11)$ & 0.7955 (9) & $0.5651(5)$ & $0.0749(18)$ \\
\hline $\mathrm{H} 15 \mathrm{~A}$ & -0.3143 & 0.7635 & 0.6159 & $0.090 *$ \\
\hline $\mathrm{C} 16$ & $-0.0009(13)$ & $0.6756(9)$ & $0.5347(6)$ & $0.080(2)$ \\
\hline H16A & -0.0068 & 0.5621 & 0.5644 & $0.095^{*}$ \\
\hline $\mathrm{C} 17$ & $0.1892(11)$ & $0.7268(8)$ & $0.4602(5)$ & $0.0665(16)$ \\
\hline H17A & 0.3134 & 0.6471 & 0.4414 & $0.080^{*}$ \\
\hline $\mathrm{C} 18$ & $0.2020(8)$ & $0.8935(7)$ & 0.4123 & $0.0465(12)$ \\
\hline C19 & $0.6721(9)$ & $0.0655(6)$ & $-0.1121(4)$ & $0.0498(13)$ \\
\hline H19A & 0.8294 & 0.0400 & -0.1523 & $0.060^{*}$ \\
\hline $\mathrm{C} 20$ & $0.5911(13)$ & $-0.0955(8)$ & $-0.0465(5)$ & $0.0728(17)$ \\
\hline $\mathrm{H} 20 \mathrm{~A}$ & 0.4515 & -0.0668 & 0.0058 & $0.087^{*}$ \\
\hline
\end{tabular}




\begin{tabular}{lllll} 
H20B & 0.7086 & -0.1390 & -0.0075 & $0.087^{*}$ \\
C21 & $0.5425(13)$ & $-0.2380(8)$ & $-0.1206(5)$ & $0.0755(19)$ \\
H21A & 0.6802 & -0.2628 & -0.1750 & $0.091^{*}$ \\
H21B & 0.5024 & -0.3453 & -0.0786 & $0.091^{*}$ \\
C22 & $0.3465(11)$ & $-0.1769(9)$ & $-0.1746(5)$ & $0.0688(18)$ \\
H22A & 0.3350 & -0.2584 & -0.2300 & $0.083^{*}$ \\
H22B & 0.2028 & -0.1782 & -0.1216 & $0.083^{*}$ \\
C23 & $0.3768(9)$ & $0.0051(8)$ & $-0.2248(4)$ & $0.0503(14)$ \\
C24 & $0.2515(10)$ & $0.0601(9)$ & $-0.3022(4)$ & $0.0624(15)$ \\
H24A & 0.1532 & -0.0175 & -0.3233 & $0.075^{*}$ \\
C25 & $0.2684(12)$ & $0.2252(10)$ & $-0.3484(5)$ & $0.079(2)$ \\
H25A & 0.1830 & 0.2591 & -0.4004 & $0.095^{*}$ \\
C26 & $0.4143(14)$ & $0.3418(9)$ & $-0.3167(6)$ & $0.086(2)$ \\
H26A & 0.4269 & 0.4550 & -0.3469 & $0.103^{*}$ \\
C27 & $0.5398(11)$ & $0.2877(8)$ & $-0.2403(5)$ & $0.0671(17)$ \\
H27A & 0.6391 & 0.3653 & -0.2199 & $0.080^{*}$ \\
C28 & $0.5226(8)$ & $0.1213(7)$ & $-0.1928(4)$ & $0.0484(13)$ \\
& & & & \\
\hline
\end{tabular}

Atomic displacement parameters $\left(\AA^{2}\right)$

\begin{tabular}{lllllll}
\hline & $U^{11}$ & $U^{22}$ & $U^{33}$ & $U^{12}$ & $U^{13}$ & $U^{23}$ \\
\hline S1 & $0.0351(7)$ & $0.0574(8)$ & $0.0551(7)$ & $-0.0009(5)$ & $-0.0088(5)$ & $-0.0201(6)$ \\
N1 & $0.045(3)$ & $0.058(3)$ & $0.049(3)$ & $0.004(2)$ & $-0.006(2)$ & $-0.026(2)$ \\
C2 & $0.049(3)$ & $0.055(3)$ & $0.047(3)$ & $-0.007(3)$ & $-0.013(2)$ & $-0.016(3)$ \\
C3 & $0.039(3)$ & $0.058(3)$ & $0.048(3)$ & $-0.002(2)$ & $-0.016(2)$ & $-0.014(3)$ \\
C4 & $0.036(3)$ & $0.071(4)$ & $0.070(4)$ & $0.000(3)$ & $-0.017(3)$ & $-0.027(3)$ \\
C5 & $0.033(3)$ & $0.075(4)$ & $0.072(4)$ & $0.005(3)$ & $-0.010(2)$ & $-0.031(3)$ \\
C6 & $0.031(3)$ & $0.050(3)$ & $0.046(3)$ & $0.004(2)$ & $-0.006(2)$ & $-0.011(2)$ \\
C7 & $0.046(3)$ & $0.057(3)$ & $0.048(3)$ & $0.010(3)$ & $-0.007(2)$ & $-0.018(3)$ \\
N8 & $0.048(3)$ & $0.059(3)$ & $0.050(3)$ & $-0.003(2)$ & $-0.008(2)$ & $-0.023(2)$ \\
C9 & $0.049(3)$ & $0.053(3)$ & $0.054(3)$ & $-0.001(3)$ & $-0.009(3)$ & $-0.022(3)$ \\
C10 & $0.102(5)$ & $0.061(4)$ & $0.060(4)$ & $-0.002(3)$ & $0.005(3)$ & $-0.015(3)$ \\
C11 & $0.109(5)$ & $0.050(4)$ & $0.070(4)$ & $0.005(3)$ & $0.010(4)$ & $-0.007(3)$ \\
C12 & $0.082(5)$ & $0.062(4)$ & $0.060(4)$ & $0.024(3)$ & $-0.015(3)$ & $-0.022(3)$ \\
C13 & $0.048(3)$ & $0.057(3)$ & $0.043(3)$ & $0.002(3)$ & $-0.012(3)$ & $-0.016(3)$ \\
C14 & $0.056(3)$ & $0.075(4)$ & $0.056(3)$ & $0.009(3)$ & $-0.009(3)$ & $-0.027(3)$ \\
C15 & $0.075(4)$ & $0.082(5)$ & $0.062(4)$ & $-0.011(4)$ & $0.006(3)$ & $-0.022(4)$ \\
C16 & $0.104(5)$ & $0.061(4)$ & $0.066(4)$ & $-0.012(4)$ & $0.001(4)$ & $-0.002(4)$ \\
C17 & $0.072(4)$ & $0.059(4)$ & $0.064(4)$ & $0.007(3)$ & $-0.003(3)$ & $-0.011(3)$ \\
C18 & $0.045(3)$ & $0.054(3)$ & $0.043(3)$ & $0.001(3)$ & $-0.012(2)$ & $-0.019(3)$ \\
C19 & $0.052(3)$ & $0.053(3)$ & $0.044(3)$ & $0.000(3)$ & $-0.007(2)$ & $-0.017(3)$ \\
C20 & $0.114(5)$ & $0.060(4)$ & $0.051(3)$ & $-0.010(4)$ & $-0.030(3)$ & $-0.011(3)$ \\
C21 & $0.119(5)$ & $0.055(4)$ & $0.056(4)$ & $-0.012(4)$ & $-0.021(4)$ & $-0.009(3)$ \\
C22 & $0.074(4)$ & $0.078(5)$ & $0.056(4)$ & $-0.028(4)$ & $-0.008(3)$ & $-0.017(3)$ \\
C23 & $0.048(3)$ & $0.057(3)$ & $0.043(3)$ & $-0.007(3)$ & $0.001(3)$ & $-0.019(3)$ \\
C24 & $0.056(3)$ & $0.079(4)$ & $0.054(3)$ & $0.003(3)$ & $-0.013(3)$ & $-0.028(3)$ \\
C25 & $0.096(5)$ & $0.084(5)$ & $0.066(4)$ & $0.026(4)$ & $-0.037(4)$ & $-0.028(4)$ \\
C26 & $0.129(6)$ & $0.057(4)$ & $0.080(5)$ & $0.016(4)$ & $-0.044(5)$ & $-0.010(4)$ \\
& & & & &
\end{tabular}




\begin{tabular}{lllllll}
$\mathrm{C} 27$ & $0.084(4)$ & $0.059(4)$ & $0.064(4)$ & $-0.003(3)$ & $-0.022(4)$ & $-0.019(3)$ \\
$\mathrm{C} 28$ & $0.050(3)$ & $0.046(3)$ & $0.049(3)$ & $0.006(2)$ & $-0.006(2)$ & $-0.018(3)$ \\
\hline
\end{tabular}

Geometric parameters $\left(A,{ }^{o}\right)$

\begin{tabular}{|c|c|c|c|}
\hline $\mathrm{S} 1-\mathrm{C} 6$ & $1.724(5)$ & $\mathrm{C} 14-\mathrm{H} 14 \mathrm{~A}$ & 0.9300 \\
\hline $\mathrm{S} 1-\mathrm{C} 3$ & $1.728(5)$ & $\mathrm{C} 15-\mathrm{C} 16$ & $1.390(9)$ \\
\hline $\mathrm{N} 1-\mathrm{C} 2$ & $1.255(6)$ & $\mathrm{C} 15-\mathrm{H} 15 \mathrm{~A}$ & 0.9300 \\
\hline $\mathrm{N} 1-\mathrm{C} 9$ & $1.471(6)$ & $\mathrm{C} 16-\mathrm{C} 17$ & $1.373(9)$ \\
\hline $\mathrm{C} 2-\mathrm{C} 3$ & $1.458(7)$ & C16-H16A & 0.9300 \\
\hline $\mathrm{C} 2-\mathrm{H} 2 \mathrm{~A}$ & 0.9300 & $\mathrm{C} 17-\mathrm{C} 18$ & $1.386(8)$ \\
\hline $\mathrm{C} 3-\mathrm{C} 4$ & $1.348(7)$ & C17-H17A & 0.9300 \\
\hline $\mathrm{C} 4-\mathrm{C} 5$ & $1.420(7)$ & $\mathrm{C} 19-\mathrm{C} 20$ & $1.496(7)$ \\
\hline $\mathrm{C} 4-\mathrm{H} 4 \mathrm{~A}$ & 0.9300 & $\mathrm{C} 19-\mathrm{C} 28$ & $1.518(7)$ \\
\hline $\mathrm{C} 5-\mathrm{C} 6$ & $1.355(6)$ & C19-H19A & 0.9800 \\
\hline C5-H5A & 0.9300 & $\mathrm{C} 20-\mathrm{C} 21$ & $1.536(8)$ \\
\hline $\mathrm{C} 6-\mathrm{C} 7$ & $1.445(7)$ & $\mathrm{C} 20-\mathrm{H} 20 \mathrm{~A}$ & 0.9700 \\
\hline $\mathrm{C} 7-\mathrm{N} 8$ & $1.263(6)$ & $\mathrm{C} 20-\mathrm{H} 20 \mathrm{~B}$ & 0.9700 \\
\hline C7-H7A & 0.9300 & $\mathrm{C} 21-\mathrm{C} 22$ & $1.508(9)$ \\
\hline $\mathrm{N} 8-\mathrm{C} 19$ & $1.479(6)$ & $\mathrm{C} 21-\mathrm{H} 21 \mathrm{~A}$ & 0.9700 \\
\hline $\mathrm{C} 9-\mathrm{C} 10$ & $1.512(8)$ & $\mathrm{C} 21-\mathrm{H} 21 \mathrm{~B}$ & 0.9700 \\
\hline $\mathrm{C} 9-\mathrm{C} 18$ & $1.520(7)$ & $\mathrm{C} 22-\mathrm{C} 23$ & $1.507(9)$ \\
\hline C9-H9A & 0.9800 & $\mathrm{C} 22-\mathrm{H} 22 \mathrm{~A}$ & 0.9700 \\
\hline $\mathrm{C} 10-\mathrm{C} 11$ & $1.522(8)$ & $\mathrm{C} 22-\mathrm{H} 22 \mathrm{~B}$ & 0.9700 \\
\hline $\mathrm{C} 10-\mathrm{H} 10 \mathrm{~A}$ & 0.9700 & $\mathrm{C} 23-\mathrm{C} 24$ & $1.384(8)$ \\
\hline $\mathrm{C} 10-\mathrm{H} 10 \mathrm{~B}$ & 0.9700 & $\mathrm{C} 23-\mathrm{C} 28$ & $1.389(7)$ \\
\hline $\mathrm{C} 11-\mathrm{C} 12$ & $1.510(9)$ & $\mathrm{C} 24-\mathrm{C} 25$ & $1.367(9)$ \\
\hline $\mathrm{C} 11-\mathrm{H} 11 \mathrm{~A}$ & 0.9700 & $\mathrm{C} 24-\mathrm{H} 24 \mathrm{~A}$ & 0.9300 \\
\hline $\mathrm{C} 11-\mathrm{H} 11 \mathrm{~B}$ & 0.9700 & $\mathrm{C} 25-\mathrm{C} 26$ & $1.390(10)$ \\
\hline $\mathrm{C} 12-\mathrm{C} 13$ & $1.509(8)$ & $\mathrm{C} 25-\mathrm{H} 25 \mathrm{~A}$ & 0.9300 \\
\hline $\mathrm{C} 12-\mathrm{H} 12 \mathrm{~A}$ & 0.9700 & $\mathrm{C} 26-\mathrm{C} 27$ & $1.374(8)$ \\
\hline $\mathrm{C} 12-\mathrm{H} 12 \mathrm{~B}$ & 0.9700 & $\mathrm{C} 26-\mathrm{H} 26 \mathrm{~A}$ & 0.9300 \\
\hline $\mathrm{C} 13-\mathrm{C} 14$ & $1.390(8)$ & $\mathrm{C} 27-\mathrm{C} 28$ & $1.382(7)$ \\
\hline $\mathrm{C} 13-\mathrm{C} 18$ & $1.398(7)$ & $\mathrm{C} 27-\mathrm{H} 27 \mathrm{~A}$ & 0.9300 \\
\hline $\mathrm{C} 14-\mathrm{C} 15$ & $1.366(9)$ & & \\
\hline $\mathrm{C} 6-\mathrm{S} 1-\mathrm{C} 3$ & $91.5(2)$ & $\mathrm{C} 16-\mathrm{C} 15-\mathrm{H} 15 \mathrm{~A}$ & 120.4 \\
\hline $\mathrm{C} 2-\mathrm{N} 1-\mathrm{C} 9$ & $116.5(4)$ & $\mathrm{C} 17-\mathrm{C} 16-\mathrm{C} 15$ & $119.2(7)$ \\
\hline $\mathrm{N} 1-\mathrm{C} 2-\mathrm{C} 3$ & $121.5(5)$ & $\mathrm{C} 17-\mathrm{C} 16-\mathrm{H} 16 \mathrm{~A}$ & 120.4 \\
\hline $\mathrm{N} 1-\mathrm{C} 2-\mathrm{H} 2 \mathrm{~A}$ & 119.3 & $\mathrm{C} 15-\mathrm{C} 16-\mathrm{H} 16 \mathrm{~A}$ & 120.4 \\
\hline $\mathrm{C} 3-\mathrm{C} 2-\mathrm{H} 2 \mathrm{~A}$ & 119.3 & $\mathrm{C} 16-\mathrm{C} 17-\mathrm{C} 18$ & $122.1(6)$ \\
\hline $\mathrm{C} 4-\mathrm{C} 3-\mathrm{C} 2$ & $129.7(5)$ & $\mathrm{C} 16-\mathrm{C} 17-\mathrm{H} 17 \mathrm{~A}$ & 119.0 \\
\hline $\mathrm{C} 4-\mathrm{C} 3-\mathrm{S} 1$ & $111.3(4)$ & $\mathrm{C} 18-\mathrm{C} 17-\mathrm{H} 17 \mathrm{~A}$ & 119.0 \\
\hline $\mathrm{C} 2-\mathrm{C} 3-\mathrm{S} 1$ & $119.1(4)$ & $\mathrm{C} 17-\mathrm{C} 18-\mathrm{C} 13$ & $118.7(5)$ \\
\hline $\mathrm{C} 3-\mathrm{C} 4-\mathrm{C} 5$ & $113.2(5)$ & $\mathrm{C} 17-\mathrm{C} 18-\mathrm{C} 9$ & $120.0(5)$ \\
\hline $\mathrm{C} 3-\mathrm{C} 4-\mathrm{H} 4 \mathrm{~A}$ & 123.4 & $\mathrm{C} 13-\mathrm{C} 18-\mathrm{C} 9$ & $121.2(5)$ \\
\hline $\mathrm{C} 5-\mathrm{C} 4-\mathrm{H} 4 \mathrm{~A}$ & 123.4 & $\mathrm{~N} 8-\mathrm{C} 19-\mathrm{C} 20$ & $109.4(4)$ \\
\hline $\mathrm{C} 6-\mathrm{C} 5-\mathrm{C} 4$ & $112.6(5)$ & $\mathrm{N} 8-\mathrm{C} 19-\mathrm{C} 28$ & $110.1(4)$ \\
\hline
\end{tabular}




\begin{tabular}{|c|c|c|c|}
\hline $\mathrm{C} 6-\mathrm{C} 5-\mathrm{H} 5 \mathrm{~A}$ & 123.7 & $\mathrm{C} 20-\mathrm{C} 19-\mathrm{C} 28$ & $113.3(4)$ \\
\hline $\mathrm{C} 4-\mathrm{C} 5-\mathrm{H} 5 \mathrm{~A}$ & 123.7 & $\mathrm{~N} 8-\mathrm{C} 19-\mathrm{H} 19 \mathrm{~A}$ & 108.0 \\
\hline $\mathrm{C} 5-\mathrm{C} 6-\mathrm{C} 7$ & $129.0(5)$ & $\mathrm{C} 20-\mathrm{C} 19-\mathrm{H} 19 \mathrm{~A}$ & 108.0 \\
\hline $\mathrm{C} 5-\mathrm{C} 6-\mathrm{S} 1$ & $111.4(4)$ & $\mathrm{C} 28-\mathrm{C} 19-\mathrm{H} 19 \mathrm{~A}$ & 108.0 \\
\hline $\mathrm{C} 7-\mathrm{C} 6-\mathrm{S} 1$ & $119.6(4)$ & $\mathrm{C} 19-\mathrm{C} 20-\mathrm{C} 21$ & $109.9(4)$ \\
\hline $\mathrm{N} 8-\mathrm{C} 7-\mathrm{C} 6$ & $121.9(5)$ & $\mathrm{C} 19-\mathrm{C} 20-\mathrm{H} 20 \mathrm{~A}$ & 109.7 \\
\hline $\mathrm{N} 8-\mathrm{C} 7-\mathrm{H} 7 \mathrm{~A}$ & 119.1 & $\mathrm{C} 21-\mathrm{C} 20-\mathrm{H} 20 \mathrm{~A}$ & 109.7 \\
\hline $\mathrm{C} 6-\mathrm{C} 7-\mathrm{H} 7 \mathrm{~A}$ & 119.1 & $\mathrm{C} 19-\mathrm{C} 20-\mathrm{H} 20 \mathrm{~B}$ & 109.7 \\
\hline $\mathrm{C} 7-\mathrm{N} 8-\mathrm{C} 19$ & $117.5(4)$ & $\mathrm{C} 21-\mathrm{C} 20-\mathrm{H} 20 \mathrm{~B}$ & 109.7 \\
\hline $\mathrm{N} 1-\mathrm{C} 9-\mathrm{C} 10$ & $109.1(4)$ & $\mathrm{H} 20 \mathrm{~A}-\mathrm{C} 20-\mathrm{H} 20 \mathrm{~B}$ & 108.2 \\
\hline $\mathrm{N} 1-\mathrm{C} 9-\mathrm{C} 18$ & $110.3(4)$ & $\mathrm{C} 22-\mathrm{C} 21-\mathrm{C} 20$ & $109.9(5)$ \\
\hline $\mathrm{C} 10-\mathrm{C} 9-\mathrm{C} 18$ & $111.6(5)$ & $\mathrm{C} 22-\mathrm{C} 21-\mathrm{H} 21 \mathrm{~A}$ & 109.7 \\
\hline $\mathrm{N} 1-\mathrm{C} 9-\mathrm{H} 9 \mathrm{~A}$ & 108.6 & $\mathrm{C} 20-\mathrm{C} 21-\mathrm{H} 21 \mathrm{~A}$ & 109.7 \\
\hline $\mathrm{C} 10-\mathrm{C} 9-\mathrm{H} 9 \mathrm{~A}$ & 108.6 & $\mathrm{C} 22-\mathrm{C} 21-\mathrm{H} 21 \mathrm{~B}$ & 109.7 \\
\hline $\mathrm{C} 18-\mathrm{C} 9-\mathrm{H} 9 \mathrm{~A}$ & 108.6 & $\mathrm{C} 20-\mathrm{C} 21-\mathrm{H} 21 \mathrm{~B}$ & 109.7 \\
\hline $\mathrm{C} 9-\mathrm{C} 10-\mathrm{C} 11$ & $109.6(5)$ & $\mathrm{H} 21 \mathrm{~A}-\mathrm{C} 21-\mathrm{H} 21 \mathrm{~B}$ & 108.2 \\
\hline $\mathrm{C} 9-\mathrm{C} 10-\mathrm{H} 10 \mathrm{~A}$ & 109.7 & $\mathrm{C} 23-\mathrm{C} 22-\mathrm{C} 21$ & $112.9(5)$ \\
\hline $\mathrm{C} 11-\mathrm{C} 10-\mathrm{H} 10 \mathrm{~A}$ & 109.7 & $\mathrm{C} 23-\mathrm{C} 22-\mathrm{H} 22 \mathrm{~A}$ & 109.0 \\
\hline $\mathrm{C} 9-\mathrm{C} 10-\mathrm{H} 10 \mathrm{~B}$ & 109.7 & $\mathrm{C} 21-\mathrm{C} 22-\mathrm{H} 22 \mathrm{~A}$ & 109.0 \\
\hline $\mathrm{C} 11-\mathrm{C} 10-\mathrm{H} 10 \mathrm{~B}$ & 109.7 & $\mathrm{C} 23-\mathrm{C} 22-\mathrm{H} 22 \mathrm{~B}$ & 109.0 \\
\hline $\mathrm{H} 10 \mathrm{~A}-\mathrm{C} 10-\mathrm{H} 10 \mathrm{~B}$ & 108.2 & $\mathrm{C} 21-\mathrm{C} 22-\mathrm{H} 22 \mathrm{~B}$ & 109.0 \\
\hline $\mathrm{C} 12-\mathrm{C} 11-\mathrm{C} 10$ & $109.6(5)$ & $\mathrm{H} 22 \mathrm{~A}-\mathrm{C} 22-\mathrm{H} 22 \mathrm{~B}$ & 107.8 \\
\hline $\mathrm{C} 12-\mathrm{C} 11-\mathrm{H} 11 \mathrm{~A}$ & 109.7 & $\mathrm{C} 24-\mathrm{C} 23-\mathrm{C} 28$ & $119.1(5)$ \\
\hline $\mathrm{C} 10-\mathrm{C} 11-\mathrm{H} 11 \mathrm{~A}$ & 109.7 & $\mathrm{C} 24-\mathrm{C} 23-\mathrm{C} 22$ & $119.5(5)$ \\
\hline $\mathrm{C} 12-\mathrm{C} 11-\mathrm{H} 11 \mathrm{~B}$ & 109.7 & $\mathrm{C} 28-\mathrm{C} 23-\mathrm{C} 22$ & $121.4(5)$ \\
\hline $\mathrm{C} 10-\mathrm{C} 11-\mathrm{H} 11 \mathrm{~B}$ & 109.7 & $\mathrm{C} 25-\mathrm{C} 24-\mathrm{C} 23$ & $121.8(6)$ \\
\hline $\mathrm{H} 11 \mathrm{~A}-\mathrm{C} 11-\mathrm{H} 11 \mathrm{~B}$ & 108.2 & $\mathrm{C} 25-\mathrm{C} 24-\mathrm{H} 24 \mathrm{~A}$ & 119.1 \\
\hline $\mathrm{C} 13-\mathrm{C} 12-\mathrm{C} 11$ & $112.9(5)$ & $\mathrm{C} 23-\mathrm{C} 24-\mathrm{H} 24 \mathrm{~A}$ & 119.1 \\
\hline $\mathrm{C} 13-\mathrm{C} 12-\mathrm{H} 12 \mathrm{~A}$ & 109.0 & $\mathrm{C} 24-\mathrm{C} 25-\mathrm{C} 26$ & $119.3(6)$ \\
\hline $\mathrm{C} 11-\mathrm{C} 12-\mathrm{H} 12 \mathrm{~A}$ & 109.0 & $\mathrm{C} 24-\mathrm{C} 25-\mathrm{H} 25 \mathrm{~A}$ & 120.4 \\
\hline $\mathrm{C} 13-\mathrm{C} 12-\mathrm{H} 12 \mathrm{~B}$ & 109.0 & $\mathrm{C} 26-\mathrm{C} 25-\mathrm{H} 25 \mathrm{~A}$ & 120.4 \\
\hline $\mathrm{C} 11-\mathrm{C} 12-\mathrm{H} 12 \mathrm{~B}$ & 109.0 & $\mathrm{C} 27-\mathrm{C} 26-\mathrm{C} 25$ & $119.1(6)$ \\
\hline $\mathrm{H} 12 \mathrm{~A}-\mathrm{C} 12-\mathrm{H} 12 \mathrm{~B}$ & 107.8 & $\mathrm{C} 27-\mathrm{C} 26-\mathrm{H} 26 \mathrm{~A}$ & 120.4 \\
\hline $\mathrm{C} 14-\mathrm{C} 13-\mathrm{C} 18$ & $118.4(5)$ & $\mathrm{C} 25-\mathrm{C} 26-\mathrm{H} 26 \mathrm{~A}$ & 120.4 \\
\hline $\mathrm{C} 14-\mathrm{C} 13-\mathrm{C} 12$ & $120.1(5)$ & $\mathrm{C} 26-\mathrm{C} 27-\mathrm{C} 28$ & $121.9(6)$ \\
\hline $\mathrm{C} 18-\mathrm{C} 13-\mathrm{C} 12$ & $121.5(5)$ & $\mathrm{C} 26-\mathrm{C} 27-\mathrm{H} 27 \mathrm{~A}$ & 119.1 \\
\hline $\mathrm{C} 15-\mathrm{C} 14-\mathrm{C} 13$ & $122.5(6)$ & $\mathrm{C} 28-\mathrm{C} 27-\mathrm{H} 27 \mathrm{~A}$ & 119.1 \\
\hline $\mathrm{C} 15-\mathrm{C} 14-\mathrm{H} 14 \mathrm{~A}$ & 118.8 & $\mathrm{C} 27-\mathrm{C} 28-\mathrm{C} 23$ & $118.8(5)$ \\
\hline $\mathrm{C} 13-\mathrm{C} 14-\mathrm{H} 14 \mathrm{~A}$ & 118.8 & $\mathrm{C} 27-\mathrm{C} 28-\mathrm{C} 19$ & $119.8(5)$ \\
\hline $\mathrm{C} 14-\mathrm{C} 15-\mathrm{C} 16$ & $119.1(6)$ & $\mathrm{C} 23-\mathrm{C} 28-\mathrm{C} 19$ & $121.3(5)$ \\
\hline $\mathrm{C} 14-\mathrm{C} 15-\mathrm{H} 15 \mathrm{~A}$ & 120.4 & & \\
\hline $\mathrm{C} 9-\mathrm{N} 1-\mathrm{C} 2-\mathrm{C} 3$ & $-176.4(5)$ & $\mathrm{C} 12-\mathrm{C} 13-\mathrm{C} 18-\mathrm{C} 17$ & $-177.8(5)$ \\
\hline $\mathrm{N} 1-\mathrm{C} 2-\mathrm{C} 3-\mathrm{C} 4$ & $172.4(6)$ & $\mathrm{C} 14-\mathrm{C} 13-\mathrm{C} 18-\mathrm{C} 9$ & $-177.3(5)$ \\
\hline $\mathrm{N} 1-\mathrm{C} 2-\mathrm{C} 3-\mathrm{S} 1$ & $-6.2(7)$ & $\mathrm{C} 12-\mathrm{C} 13-\mathrm{C} 18-\mathrm{C} 9$ & $5.4(7)$ \\
\hline $\mathrm{C} 6-\mathrm{S} 1-\mathrm{C} 3-\mathrm{C} 4$ & $-1.4(5)$ & $\mathrm{N} 1-\mathrm{C} 9-\mathrm{C} 18-\mathrm{C} 17$ & $39.8(6)$ \\
\hline $\mathrm{C} 6-\mathrm{S} 1-\mathrm{C} 3-\mathrm{C} 2$ & $177.5(4)$ & $\mathrm{C} 10-\mathrm{C} 9-\mathrm{C} 18-\mathrm{C} 17$ & $161.1(5)$ \\
\hline $\mathrm{C} 2-\mathrm{C} 3-\mathrm{C} 4-\mathrm{C} 5$ & $-177.8(5)$ & $\mathrm{N} 1-\mathrm{C} 9-\mathrm{C} 18-\mathrm{C} 13$ & $-143.5(4)$ \\
\hline
\end{tabular}




$\begin{array}{llll}\mathrm{S} 1-\mathrm{C} 3-\mathrm{C} 4-\mathrm{C} 5 & 0.9(6) & \mathrm{C} 10-\mathrm{C} 9-\mathrm{C} 18-\mathrm{C} 13 & -22.1(6) \\ \mathrm{C} 3-\mathrm{C} 4-\mathrm{C} 5-\mathrm{C} 6 & 0.2(7) & \mathrm{C} 7-\mathrm{N} 8-\mathrm{C} 19-\mathrm{C} 20 & 105.5(6) \\ \mathrm{C} 4-\mathrm{C} 5-\mathrm{C} 6-\mathrm{C} 7 & 178.9(5) & \mathrm{C} 7-\mathrm{N} 8-\mathrm{C} 19-\mathrm{C} 28 & -129.5(5) \\ \mathrm{C} 4-\mathrm{C} 5-\mathrm{C} 6-\mathrm{S} 1 & -1.3(6) & \mathrm{N} 8-\mathrm{C} 19-\mathrm{C} 20-\mathrm{C} 21 & 170.8(5) \\ \mathrm{C} 3-\mathrm{S} 1-\mathrm{C} 6-\mathrm{C} 5 & 1.5(4) & \mathrm{C} 28-\mathrm{C} 19-\mathrm{C} 20-\mathrm{C} 21 & 47.7(7) \\ \mathrm{C} 3-\mathrm{S} 1-\mathrm{C} 6-\mathrm{C} 7 & -178.7(4) & \mathrm{C} 19-\mathrm{C} 20-\mathrm{C} 21-\mathrm{C} 22 & -64.0(7) \\ \mathrm{C} 5-\mathrm{C} 6-\mathrm{C} 7-\mathrm{N} 8 & -179.1(6) & \mathrm{C} 20-\mathrm{C} 21-\mathrm{C} 22-\mathrm{C} 23 & 49.0(7) \\ \mathrm{S} 1-\mathrm{C} 6-\mathrm{C} 7-\mathrm{N} 8 & 1.1(7) & \mathrm{C} 21-\mathrm{C} 22-\mathrm{C} 23-\mathrm{C} 24 & 161.6(5) \\ \mathrm{C} 6-\mathrm{C} 7-\mathrm{N} 8-\mathrm{C} 19 & -178.2(5) & \mathrm{C} 21-\mathrm{C} 22-\mathrm{C} 23-\mathrm{C} 28 & -20.5(8) \\ \mathrm{C} 2-\mathrm{N} 1-\mathrm{C} 9-\mathrm{C} 10 & 102.3(6) & \mathrm{C} 28-\mathrm{C} 23-\mathrm{C} 24-\mathrm{C} 25 & 0.4(8) \\ \mathrm{C} 2-\mathrm{N} 1-\mathrm{C} 9-\mathrm{C} 18 & -134.9(5) & \mathrm{C} 22-\mathrm{C} 23-\mathrm{C} 24-\mathrm{C} 25 & 178.4(6) \\ \mathrm{N} 1-\mathrm{C} 9-\mathrm{C} 10-\mathrm{C} 11 & 173.7(5) & \mathrm{C} 23-\mathrm{C} 24-\mathrm{C} 25-\mathrm{C} 26 & -0.3(9) \\ \mathrm{C} 18-\mathrm{C} 9-\mathrm{C} 10-\mathrm{C} 11 & 51.6(7) & \mathrm{C} 24-\mathrm{C} 25-\mathrm{C} 26-\mathrm{C} 27 & 0.5(10) \\ \mathrm{C} 9-\mathrm{C} 10-\mathrm{C} 11-\mathrm{C} 12 & -66.2(7) & \mathrm{C} 25-\mathrm{C} 26-\mathrm{C} 27-\mathrm{C} 28 & -0.9(10) \\ \mathrm{C} 10-\mathrm{C} 11-\mathrm{C} 12-\mathrm{C} 13 & 48.1(7) & \mathrm{C} 26-\mathrm{C} 27-\mathrm{C} 28-\mathrm{C} 23 & 1.0(8) \\ \mathrm{C} 11-\mathrm{C} 12-\mathrm{C} 13-\mathrm{C} 14 & 164.1(5) & \mathrm{C} 26-\mathrm{C} 27-\mathrm{C} 28-\mathrm{C} 19 & 177.5(6) \\ \mathrm{C} 11-\mathrm{C} 12-\mathrm{C} 13-\mathrm{C} 18 & -18.7(8) & \mathrm{C} 24-\mathrm{C} 23-\mathrm{C} 28-\mathrm{C} 27 & -0.7(7) \\ \mathrm{C} 18-\mathrm{C} 13-\mathrm{C} 14-\mathrm{C} 15 & -0.5(8) & \mathrm{C} 22-\mathrm{C} 23-\mathrm{C} 28-\mathrm{C} 27 & -178.7(6) \\ \mathrm{C} 12-\mathrm{C} 13-\mathrm{C} 14-\mathrm{C} 15 & 176.8(5) & \mathrm{C} 24-\mathrm{C} 23-\mathrm{C} 28-\mathrm{C} 19 & -177.2(5) \\ \mathrm{C} 13-\mathrm{C} 14-\mathrm{C} 15-\mathrm{C} 16 & 0.5(9) & \mathrm{C} 22-\mathrm{C} 23-\mathrm{C} 28-\mathrm{C} 19 & 4.9(8) \\ \mathrm{C} 14-\mathrm{C} 15-\mathrm{C} 16-\mathrm{C} 17 & 0.5(10) & \mathrm{N} 8-\mathrm{C} 19-\mathrm{C} 28-\mathrm{C} 27 & 41.7(6) \\ \mathrm{C} 15-\mathrm{C} 16-\mathrm{C} 17-\mathrm{C} 18 & -1.6(10) & \mathrm{C} 20-\mathrm{C} 19-\mathrm{C} 28-\mathrm{C} 27 & 164.5(5) \\ \mathrm{C} 16-\mathrm{C} 17-\mathrm{C} 18-\mathrm{C} 13 & 1.6(9) & \mathrm{N} 8-\mathrm{C} 19-\mathrm{C} 28-\mathrm{C} 23 & -141.9(5) \\ \mathrm{C} 16-\mathrm{C} 17-\mathrm{C} 18-\mathrm{C} 9 & 178.4(6) & \mathrm{C} 20-\mathrm{C} 19-\mathrm{C} 28-\mathrm{C} 23 & -19.1(7) \\ \mathrm{C} 14-\mathrm{C} 13-\mathrm{C} 18-\mathrm{C} 17 & -0.5(7) & & \end{array}$

Hydrogen-bond geometry $\left(\AA,{ }^{\circ}\right)$

\begin{tabular}{lllll}
\hline$D-\mathrm{H} \cdots A$ & $D-\mathrm{H}$ & $\mathrm{H} \cdots A$ & $D \cdots A$ & $D-\mathrm{H} \cdots A$ \\
\hline $\mathrm{C} 4-\mathrm{H} 4 A \cdots \mathrm{S} 1^{\mathrm{i}}$ & 0.93 & 3.00 & $3.562(5)$ & 121 \\
$\mathrm{C} 5-\mathrm{H} 5 A \cdots \mathrm{S} 1^{\mathrm{i}}$ & 0.93 & 2.97 & $3.547(5)$ & 122 \\
\hline
\end{tabular}

Symmetry code: (i) $x+1, y, z$.

(II) 2,5-Bis\{[(R)-(-)-1-(4-methoxyphenyl)ethyl] iminomethyl\}thiophene

\section{Crystal data}

$\mathrm{C}_{24} \mathrm{H}_{26} \mathrm{~N}_{2} \mathrm{O}_{2} \mathrm{~S}$

$M_{r}=406.53$

Monoclinic, $C 2$

$a=25.3917(13) \AA$

$b=5.9488(3) \AA$

$c=7.5623(4) \AA$

$\beta=97.174(4)^{\circ}$

$V=1133.34(10) \AA^{3}$

$Z=2$

$F(000)=432$
$D_{\mathrm{x}}=1.191 \mathrm{Mg} \mathrm{m}^{-3}$

Melting point: $405 \mathrm{~K}$

Mo $K \alpha$ radiation, $\lambda=0.71073 \AA$

Cell parameters from 2504 reflections

$\theta=3.0-24.2^{\circ}$

$\mu=0.16 \mathrm{~mm}^{-1}$

$T=298 \mathrm{~K}$

Prism, colourless

$0.45 \times 0.33 \times 0.12 \mathrm{~mm}$ 


\section{Data collection}

Agilent Xcalibur (Atlas, Gemini) diffractometer

Radiation source: Enhance (Mo) X-ray Source Graphite monochromator

$\omega$ scans

Absorption correction: analytical

(CrysAlis PRO; Agilent, 2013)

$T_{\min }=0.973, T_{\max }=0.993$

\section{Refinement}

Refinement on $F^{2}$

Least-squares matrix: full

$R\left[F^{2}>2 \sigma\left(F^{2}\right)\right]=0.036$

$w R\left(F^{2}\right)=0.085$

$S=1.02$

2221 reflections

134 parameters

1 restraint

Hydrogen site location: inferred from

neighbouring sites
6341 measured reflections

2221 independent reflections

1892 reflections with $I>2 \sigma(I)$

$R_{\text {int }}=0.027$

$\theta_{\text {max }}=26.1^{\circ}, \theta_{\text {min }}=3.0^{\circ}$

$h=-31 \rightarrow 31$

$k=-7 \rightarrow 7$

$l=-9 \rightarrow 9$

$\mathrm{H}$-atom parameters constrained

$w=1 /\left[\sigma^{2}\left(F_{\mathrm{o}}^{2}\right)+(0.0393 P)^{2}+0.1801 P\right]$

where $P=\left(F_{\mathrm{o}}^{2}+2 F_{\mathrm{c}}^{2}\right) / 3$

$(\Delta / \sigma)_{\max }<0.001$

$\Delta \rho_{\max }=0.11$ e $\AA^{-3}$

$\Delta \rho_{\min }=-0.17$ e $\AA^{-3}$

Absolute structure: Flack $x$ determined using 708 quotients $\left[\left(I^{+}\right)-\left(I^{-}\right)\right] /\left[\left(I^{+}\right)+\left(I^{-}\right)\right]$(Parsons et al. 2013)

Absolute structure parameter: -0.02 (4)

Fractional atomic coordinates and isotropic or equivalent isotropic displacement parameters $\left(\AA^{2}\right)$

\begin{tabular}{|c|c|c|c|c|}
\hline & $x$ & $y$ & $z$ & $U_{\text {iso }} * / U_{\text {eq }}$ \\
\hline $\mathrm{S} 1$ & 0.5000 & $0.37429(14)$ & 0.5000 & 0.0490 \\
\hline N1 & $0.56565(9)$ & $0.3213(4)$ & 0.1855 & $0.0471(6)$ \\
\hline $\mathrm{C} 2$ & $0.55195(11)$ & $0.5176(5)$ & $0.2189(4)$ & $0.0469(7)$ \\
\hline $\mathrm{H} 2 \mathrm{~A}$ & 0.5608 & 0.6324 & 0.1445 & $0.056 *$ \\
\hline $\mathrm{C} 3$ & $0.52314(10)$ & $0.5774(5)$ & $0.3665(4)$ & $0.0449(6)$ \\
\hline $\mathrm{C} 4$ & $0.51313(13)$ & $0.7856(5)$ & $0.4229(4)$ & $0.0596(8)$ \\
\hline $\mathrm{H} 4 \mathrm{~A}$ & 0.5225 & 0.9159 & 0.3665 & $0.072 *$ \\
\hline $\mathrm{C} 5$ & $0.59848(11)$ & $0.2933(4)$ & $0.0386(4)$ & $0.0484(7)$ \\
\hline H5A & 0.5949 & 0.4291 & -0.0354 & $0.058^{*}$ \\
\hline C6 & $0.57863(13)$ & $0.0963(6)$ & $-0.0754(4)$ & $0.0658(8)$ \\
\hline H6A & 0.5416 & 0.1162 & -0.1164 & $0.099 *$ \\
\hline H6B & 0.5835 & -0.0394 & -0.0065 & $0.099 *$ \\
\hline $\mathrm{H} 6 \mathrm{C}$ & 0.5981 & 0.0861 & -0.1759 & $0.099 *$ \\
\hline $\mathrm{C} 7$ & $0.65613(11)$ & $0.2719(4)$ & 0.1189 & $0.0441(6)$ \\
\hline $\mathrm{C} 8$ & $0.69277(11)$ & $0.4349(4)$ & $0.0871(4)$ & $0.0494(7)$ \\
\hline H8A & 0.6817 & 0.5585 & 0.0168 & $0.059 *$ \\
\hline $\mathrm{C} 9$ & $0.74515(11)$ & $0.4171(5)$ & $0.1576(4)$ & $0.0571(8)$ \\
\hline H9A & 0.7692 & 0.5278 & 0.1343 & $0.069 *$ \\
\hline $\mathrm{C} 10$ & $0.76215(11)$ & $0.2354(6)$ & $0.2628(4)$ & $0.0546(7)$ \\
\hline $\mathrm{C} 11$ & $0.72642(12)$ & $0.0745(6)$ & $0.2994(4)$ & $0.0593(8)$ \\
\hline H11A & 0.7375 & -0.0469 & 0.3722 & $0.071 *$ \\
\hline $\mathrm{C} 12$ & $0.67374(12)$ & $0.0936(6)$ & 0.2275 & $0.0551(8)$ \\
\hline $\mathrm{H} 12 \mathrm{~A}$ & 0.6497 & -0.0161 & 0.2528 & $0.066^{*}$ \\
\hline O1 & $0.81556(9)$ & $0.2307(5)$ & $0.3227(3)$ & $0.0781(7)$ \\
\hline $\mathrm{C} 13$ & $0.83539(15)$ & $0.0345(9)$ & $0.4189(5)$ & $0.1010(15)$ \\
\hline
\end{tabular}




\begin{tabular}{lllll} 
H13A & 0.8734 & 0.0434 & 0.4432 & $0.152^{*}$ \\
H13B & 0.8257 & -0.0970 & 0.3487 & $0.152^{*}$ \\
H13C & 0.8204 & 0.0258 & 0.5292 & $0.152^{*}$ \\
\hline
\end{tabular}

Atomic displacement parameters $\left(\AA^{2}\right)$

\begin{tabular}{lllllll}
\hline & $U^{11}$ & $U^{22}$ & $U^{33}$ & $U^{12}$ & $U^{13}$ & $U^{23}$ \\
\hline S1 & $0.0540(6)$ & $0.0354(5)$ & $0.0603(6)$ & 0.000 & $0.0183(4)$ & 0.000 \\
N1 & $0.0442(13)$ & $0.0512(16)$ & $0.0477(13)$ & $0.0014(10)$ & $0.0129(10)$ & $0.0058(10)$ \\
C2 & $0.0460(15)$ & $0.0443(18)$ & $0.0507(16)$ & $-0.0021(13)$ & $0.0071(13)$ & $0.0121(13)$ \\
C3 & $0.0421(14)$ & $0.0392(14)$ & $0.0534(17)$ & $-0.0002(12)$ & $0.0062(13)$ & $0.0052(12)$ \\
C4 & $0.074(2)$ & $0.0365(16)$ & $0.071(2)$ & $0.0007(13)$ & $0.0193(16)$ & $0.0074(13)$ \\
C5 & $0.0497(16)$ & $0.0537(18)$ & $0.0434(15)$ & $0.0015(12)$ & $0.0120(12)$ & $0.0097(12)$ \\
C6 & $0.0626(19)$ & $0.079(2)$ & $0.0551(19)$ & $-0.0010(18)$ & $0.0058(15)$ & $-0.0036(17)$ \\
C7 & $0.0468(15)$ & $0.0492(15)$ & $0.0387(14)$ & $0.0022(13)$ & $0.0147(12)$ & $0.0018(12)$ \\
C8 & $0.0559(17)$ & $0.0500(17)$ & $0.0451(14)$ & $-0.0025(13)$ & $0.0169(13)$ & $0.0045(12)$ \\
C9 & $0.0528(17)$ & $0.067(2)$ & $0.0548(17)$ & $-0.0139(16)$ & $0.0177(14)$ & $-0.0004(16)$ \\
C10 & $0.0455(16)$ & $0.078(2)$ & $0.0414(16)$ & $0.0001(15)$ & $0.0080(13)$ & $-0.0080(15)$ \\
C11 & $0.0575(18)$ & $0.068(2)$ & $0.0524(18)$ & $0.0087(17)$ & $0.0086(14)$ & $0.0150(15)$ \\
C12 & $0.0527(17)$ & $0.0569(17)$ & $0.0574(19)$ & $-0.0031(14)$ & $0.0138(14)$ & $0.0147(15)$ \\
O1 & $0.0478(13)$ & $0.121(2)$ & $0.0642(14)$ & $-0.0021(14)$ & $0.0011(10)$ & $-0.0011(14)$ \\
C13 & $0.063(2)$ & $0.161(4)$ & $0.075(3)$ & $0.022(3)$ & $-0.0086(19)$ & $0.022(3)$ \\
& & & & & &
\end{tabular}

Geometric parameters $\left(\AA,{ }^{\circ}\right)$

\begin{tabular}{llll}
\hline $\mathrm{S} 1-\mathrm{C} 3^{\mathrm{i}}$ & $1.724(3)$ & $\mathrm{C} 7-\mathrm{C} 12$ & $1.381(4)$ \\
$\mathrm{S} 1-\mathrm{C} 3$ & $1.724(3)$ & $\mathrm{C} 7-\mathrm{C} 8$ & $1.385(3)$ \\
$\mathrm{N} 1-\mathrm{C} 2$ & $1.253(3)$ & $\mathrm{C} 8-\mathrm{C} 9$ & $1.374(4)$ \\
$\mathrm{N} 1-\mathrm{C} 5$ & $1.480(3)$ & $\mathrm{C} 8-\mathrm{H} 8 \mathrm{~A}$ & 0.9300 \\
$\mathrm{C} 2-\mathrm{C} 3$ & $1.453(4)$ & $\mathrm{C} 9-\mathrm{C} 10$ & $1.379(4)$ \\
$\mathrm{C} 2-\mathrm{H} 2 \mathrm{~A}$ & 0.9300 & $\mathrm{C} 9-\mathrm{H} 9 \mathrm{~A}$ & 0.9300 \\
$\mathrm{C} 3-\mathrm{C} 4$ & $1.345(4)$ & $\mathrm{C} 10-\mathrm{C} 11$ & $1.371(4)$ \\
$\mathrm{C} 4-\mathrm{C} 4$ & $1.413(6)$ & $\mathrm{C} 10-\mathrm{O} 1$ & $1.375(3)$ \\
$\mathrm{C} 4-\mathrm{H} 4 \mathrm{~A}$ & 0.9300 & $\mathrm{C} 11-\mathrm{C} 12$ & $1.384(4)$ \\
$\mathrm{C} 5-\mathrm{C} 6$ & $1.504(4)$ & $\mathrm{C} 11-\mathrm{H} 11 \mathrm{~A}$ & 0.9300 \\
$\mathrm{C} 5-\mathrm{C} 7$ & $1.519(4)$ & $\mathrm{C} 12-\mathrm{H} 12 \mathrm{~A}$ & 0.9300 \\
$\mathrm{C} 5-\mathrm{H} 5 \mathrm{~A}$ & 0.9800 & $\mathrm{O} 1-\mathrm{C} 13$ & $1.433(5)$ \\
$\mathrm{C} 6-\mathrm{H} 6 \mathrm{~A}$ & 0.9600 & $\mathrm{C} 13-\mathrm{H} 13 \mathrm{~A}$ & 0.9600 \\
$\mathrm{C} 6-\mathrm{H} 6 \mathrm{~B}$ & 0.9600 & $\mathrm{C} 13-\mathrm{H} 13 \mathrm{~B}$ & 0.9600 \\
$\mathrm{C} 6-\mathrm{H} 6 \mathrm{C}$ & 0.9600 & $\mathrm{C} 13-\mathrm{H} 13 \mathrm{C}$ & 0.9600 \\
& & & $121.8(2)$ \\
$\mathrm{C} 3-\mathrm{S} 1-\mathrm{C} 3$ & $91.01(19)$ & $\mathrm{C} 12-\mathrm{C} 7-\mathrm{C} 5$ & $120.4(2)$ \\
$\mathrm{C} 2-\mathrm{N} 1-\mathrm{C} 5$ & $116.9(2)$ & $\mathrm{C} 8-\mathrm{C} 7-\mathrm{C} 5$ & $121.2(3)$ \\
$\mathrm{N} 1-\mathrm{C} 2-\mathrm{C} 3$ & $124.2(3)$ & $\mathrm{C} 9-\mathrm{C} 8-\mathrm{C} 7$ & 119.4 \\
$\mathrm{~N} 1-\mathrm{C} 2-\mathrm{H} 2 \mathrm{~A}$ & 117.9 & $\mathrm{C} 9-\mathrm{C} 8-\mathrm{H} 8 \mathrm{~A}$ & 119.4 \\
$\mathrm{C} 3-\mathrm{C} 2-\mathrm{H} 2 \mathrm{~A}$ & 117.9 & $\mathrm{C} 7-\mathrm{C} 8-\mathrm{H} 8 \mathrm{~A}$ & $120.1(3)$ \\
$\mathrm{C} 4-\mathrm{C} 3-\mathrm{C} 2$ & $127.1(3)$ & $\mathrm{C} 8-\mathrm{C} 9-\mathrm{C} 10$ & 119.9 \\
$\mathrm{C} 4-\mathrm{C} 3-\mathrm{S} 1$ & $111.6(2)$ & $\mathrm{C} 8-\mathrm{C} 9-\mathrm{H} 9 \mathrm{~A}$ &
\end{tabular}




\begin{tabular}{|c|c|c|c|}
\hline $\mathrm{C} 2-\mathrm{C} 3-\mathrm{S} 1$ & $121.3(2)$ & $\mathrm{C} 10-\mathrm{C} 9-\mathrm{H} 9 \mathrm{~A}$ & 119.9 \\
\hline $\mathrm{C} 3-\mathrm{C} 4-\mathrm{C}^{\mathrm{i}}$ & $112.91(17)$ & $\mathrm{C} 11-\mathrm{C} 10-\mathrm{O} 1$ & $124.7(3)$ \\
\hline $\mathrm{C} 3-\mathrm{C} 4-\mathrm{H} 4 \mathrm{~A}$ & 123.5 & $\mathrm{C} 11-\mathrm{C} 10-\mathrm{C} 9$ & $119.8(3)$ \\
\hline $\mathrm{C} 4-\mathrm{C} 4-\mathrm{H} 4 \mathrm{~A}$ & 123.5 & $\mathrm{O} 1-\mathrm{C} 10-\mathrm{C} 9$ & $115.5(3)$ \\
\hline $\mathrm{N} 1-\mathrm{C} 5-\mathrm{C} 6$ & $109.7(2)$ & $\mathrm{C} 10-\mathrm{C} 11-\mathrm{C} 12$ & $119.7(3)$ \\
\hline $\mathrm{N} 1-\mathrm{C} 5-\mathrm{C} 7$ & $108.3(2)$ & $\mathrm{C} 10-\mathrm{C} 11-\mathrm{H} 11 \mathrm{~A}$ & 120.2 \\
\hline $\mathrm{C} 6-\mathrm{C} 5-\mathrm{C} 7$ & $113.7(2)$ & $\mathrm{C} 12-\mathrm{C} 11-\mathrm{H} 11 \mathrm{~A}$ & 120.2 \\
\hline $\mathrm{N} 1-\mathrm{C} 5-\mathrm{H} 5 \mathrm{~A}$ & 108.3 & $\mathrm{C} 7-\mathrm{C} 12-\mathrm{C} 11$ & $121.5(3)$ \\
\hline $\mathrm{C} 6-\mathrm{C} 5-\mathrm{H} 5 \mathrm{~A}$ & 108.3 & $\mathrm{C} 7-\mathrm{C} 12-\mathrm{H} 12 \mathrm{~A}$ & 119.3 \\
\hline $\mathrm{C} 7-\mathrm{C} 5-\mathrm{H} 5 \mathrm{~A}$ & 108.3 & $\mathrm{C} 11-\mathrm{C} 12-\mathrm{H} 12 \mathrm{~A}$ & 119.3 \\
\hline $\mathrm{C} 5-\mathrm{C} 6-\mathrm{H} 6 \mathrm{~A}$ & 109.5 & $\mathrm{C} 10-\mathrm{O} 1-\mathrm{C} 13$ & $117.0(3)$ \\
\hline $\mathrm{C} 5-\mathrm{C} 6-\mathrm{H} 6 \mathrm{~B}$ & 109.5 & $\mathrm{O} 1-\mathrm{C} 13-\mathrm{H} 13 \mathrm{~A}$ & 109.5 \\
\hline $\mathrm{H} 6 \mathrm{~A}-\mathrm{C} 6-\mathrm{H} 6 \mathrm{~B}$ & 109.5 & $\mathrm{O} 1-\mathrm{C} 13-\mathrm{H} 13 \mathrm{~B}$ & 109.5 \\
\hline $\mathrm{C} 5-\mathrm{C} 6-\mathrm{H} 6 \mathrm{C}$ & 109.5 & $\mathrm{H} 13 \mathrm{~A}-\mathrm{C} 13-\mathrm{H} 13 \mathrm{~B}$ & 109.5 \\
\hline $\mathrm{H} 6 \mathrm{~A}-\mathrm{C} 6-\mathrm{H} 6 \mathrm{C}$ & 109.5 & $\mathrm{O} 1-\mathrm{C} 13-\mathrm{H} 13 \mathrm{C}$ & 109.5 \\
\hline $\mathrm{H} 6 \mathrm{~B}-\mathrm{C} 6-\mathrm{H} 6 \mathrm{C}$ & 109.5 & $\mathrm{H} 13 \mathrm{~A}-\mathrm{C} 13-\mathrm{H} 13 \mathrm{C}$ & 109.5 \\
\hline $\mathrm{C} 12-\mathrm{C} 7-\mathrm{C} 8$ & $117.8(3)$ & $\mathrm{H} 13 \mathrm{~B}-\mathrm{C} 13-\mathrm{H} 13 \mathrm{C}$ & 109.5 \\
\hline $\mathrm{C} 5-\mathrm{N} 1-\mathrm{C} 2-\mathrm{C} 3$ & $-175.3(2)$ & $\mathrm{C} 12-\mathrm{C} 7-\mathrm{C} 8-\mathrm{C} 9$ & $1.5(4)$ \\
\hline $\mathrm{N} 1-\mathrm{C} 2-\mathrm{C} 3-\mathrm{C} 4$ & $171.0(3)$ & $\mathrm{C} 5-\mathrm{C} 7-\mathrm{C} 8-\mathrm{C} 9$ & $-179.4(3)$ \\
\hline $\mathrm{N} 1-\mathrm{C} 2-\mathrm{C} 3-\mathrm{S} 1$ & $-5.7(4)$ & $\mathrm{C} 7-\mathrm{C} 8-\mathrm{C} 9-\mathrm{C} 10$ & $-0.3(4)$ \\
\hline $\mathrm{C} 33^{\mathrm{i}}-\mathrm{S} 1-\mathrm{C} 3-\mathrm{C} 4$ & $-0.22(17)$ & $\mathrm{C} 8-\mathrm{C} 9-\mathrm{C} 10-\mathrm{C} 11$ & $-1.1(4)$ \\
\hline $\mathrm{C} 33^{\mathrm{i}}-\mathrm{S} 1-\mathrm{C} 3-\mathrm{C} 2$ & $176.9(3)$ & $\mathrm{C} 8-\mathrm{C} 9-\mathrm{C} 10-\mathrm{O} 1$ & $178.3(2)$ \\
\hline $\mathrm{C} 2-\mathrm{C} 3-\mathrm{C} 4-\mathrm{C}^{\mathrm{i}}$ & $-176.3(3)$ & $\mathrm{O} 1-\mathrm{C} 10-\mathrm{C} 11-\mathrm{C} 12$ & $-178.1(3)$ \\
\hline $\mathrm{S} 1-\mathrm{C} 3-\mathrm{C} 4-\mathrm{C} 4^{\mathrm{i}}$ & $0.6(4)$ & $\mathrm{C} 9-\mathrm{C} 10-\mathrm{C} 11-\mathrm{C} 12$ & $1.2(4)$ \\
\hline $\mathrm{C} 2-\mathrm{N} 1-\mathrm{C} 5-\mathrm{C} 6$ & $-136.4(3)$ & $\mathrm{C} 8-\mathrm{C} 7-\mathrm{C} 12-\mathrm{C} 11$ & $-1.4(4)$ \\
\hline $\mathrm{C} 2-\mathrm{N} 1-\mathrm{C} 5-\mathrm{C} 7$ & $99.0(3)$ & $\mathrm{C} 5-\mathrm{C} 7-\mathrm{C} 12-\mathrm{C} 11$ & $179.6(3)$ \\
\hline $\mathrm{N} 1-\mathrm{C} 5-\mathrm{C} 7-\mathrm{C} 12$ & $63.9(3)$ & $\mathrm{C} 10-\mathrm{C} 11-\mathrm{C} 12-\mathrm{C} 7$ & $0.0(5)$ \\
\hline $\mathrm{C} 6-\mathrm{C} 5-\mathrm{C} 7-\mathrm{C} 12$ & $-58.3(3)$ & $\mathrm{C} 11-\mathrm{C} 10-\mathrm{O} 1-\mathrm{C} 13$ & $4.8(4)$ \\
\hline $\mathrm{N} 1-\mathrm{C} 5-\mathrm{C} 7-\mathrm{C} 8$ & $-115.0(3)$ & $\mathrm{C} 9-\mathrm{C} 10-\mathrm{O} 1-\mathrm{C} 13$ & $-174.6(3)$ \\
\hline $\mathrm{C} 6-\mathrm{C} 5-\mathrm{C} 7-\mathrm{C} 8$ & $122.7(3)$ & & \\
\hline
\end{tabular}

Symmetry code: (i) $-x+1, y,-z+1$.

Hydrogen-bond geometry $\left(\AA,{ }^{\circ}\right)$

\begin{tabular}{lllll}
\hline$D-\mathrm{H} \cdots A$ & $D-\mathrm{H}$ & $\mathrm{H} \cdots A$ & $D \cdots A$ & $D-\mathrm{H} \cdots A$ \\
\hline $\mathrm{C} 4-\mathrm{H} 4 A \cdots \mathrm{S} 1^{\mathrm{ii}}$ & 0.93 & 2.99 & $3.572(3)$ & 122 \\
\hline
\end{tabular}

Symmetry code: (ii) $x, y+1, z$.

(III) 2,5-Bis $\{[(R)-(-)-1-(4-f l u o r o p h e n y l) e t h y l]$ iminomethyl\}thiophene

\section{Crystal data}

$\mathrm{C}_{22} \mathrm{H}_{20} \mathrm{~F}_{2} \mathrm{~N}_{2} \mathrm{~S}$

$M_{r}=382.46$

Orthorhombic, $P 2{ }_{1} 2_{1} 2$

$a=21.1153(16) \AA$

$b=7.7846(6) \AA$

$c=6.1343(5) \AA$

$V=1008.32(14) \AA^{3}$
$Z=2$

$F(000)=400$

$D_{\mathrm{x}}=1.260 \mathrm{Mg} \mathrm{m}^{-3}$

Melting point: $420 \mathrm{~K}$

Mo $K \alpha$ radiation, $\lambda=0.71073 \AA$

Cell parameters from 2744 reflections

$\theta=3.8-23.2^{\circ}$ 
$\mu=0.19 \mathrm{~mm}^{-1}$

$T=298 \mathrm{~K}$

Data collection

Agilent Xcalibur (Atlas, Gemini) diffractometer

Radiation source: Enhance (Mo) X-ray Source

Graphite monochromator

Detector resolution: 10.5564 pixels $\mathrm{mm}^{-1}$

$\omega$ scans

Absorption correction: analytical

CrysAlis PRO, (Agilent, 2013)

$T_{\min }=0.904, T_{\max }=0.958$

\section{Refinement}

Refinement on $F^{2}$

Least-squares matrix: full

$R\left[F^{2}>2 \sigma\left(F^{2}\right)\right]=0.044$

$w R\left(F^{2}\right)=0.092$

$S=1.06$

2067 reflections

124 parameters

0 restraints

Primary atom site location: structure-invariant direct methods

Secondary atom site location: difference Fourier map
Prism, colourless

$0.89 \times 0.47 \times 0.33 \mathrm{~mm}$

12336 measured reflections

2067 independent reflections

1591 reflections with $I>2 \sigma(I)$

$R_{\text {int }}=0.058$

$\theta_{\text {max }}=26.4^{\circ}, \theta_{\text {min }}=3.8^{\circ}$

$h=-26 \rightarrow 26$

$k=-9 \rightarrow 9$

$l=-7 \rightarrow 7$

Hydrogen site location: inferred from neighbouring sites

$\mathrm{H}$-atom parameters constrained

$w=1 /\left[\sigma^{2}\left(F_{\mathrm{o}}^{2}\right)+(0.0384 P)^{2}+0.0613 P\right]$

where $P=\left(F_{\mathrm{o}}^{2}+2 F_{\mathrm{c}}{ }^{2}\right) / 3$

$(\Delta / \sigma)_{\max }<0.001$

$\Delta \rho_{\max }=0.15$ e $\AA^{-3}$

$\Delta \rho_{\text {min }}=-0.25$ e $\AA^{-3}$

Absolute structure: Flack $x$ determined using 518 quotients $\left[\left(I^{+}\right)-\left(I^{-}\right)\right] /\left[\left(I^{+}\right)+\left(I^{-}\right)\right]$(Parsons et al. 2013)

Absolute structure parameter: 0.07 (6)

Fractional atomic coordinates and isotropic or equivalent isotropic displacement parameters $\left(\AA^{2}\right)$

\begin{tabular}{|c|c|c|c|c|}
\hline & $x$ & $y$ & $z$ & $U_{\text {iso }} * / U_{\text {eq }}$ \\
\hline $\mathrm{S} 1$ & 0.5000 & 0.0000 & $1.06817(15)$ & 0.0479 \\
\hline $\mathrm{F} 1$ & $0.85802(10)$ & 0.3203 & $0.5731(5)$ & $0.1163(9)$ \\
\hline N1 & $0.58698(11)$ & 0.3119 & $1.0120(4)$ & $0.0498(6)$ \\
\hline $\mathrm{C} 2$ & $0.56853(13)$ & $0.2830(4)$ & $1.2046(5)$ & $0.0479(8)$ \\
\hline $\mathrm{H} 2 \mathrm{~A}$ & 0.5795 & 0.3610 & 1.3130 & $0.057^{*}$ \\
\hline $\mathrm{C} 3$ & $0.53102(13)$ & $0.1344(4)$ & $1.2646(4)$ & $0.0461(8)$ \\
\hline $\mathrm{C} 4$ & $0.51751(14)$ & $0.0774(4)$ & $1.4690(4)$ & $0.0556(8)$ \\
\hline H4A & 0.5300 & 0.1342 & 1.5953 & $0.067 *$ \\
\hline $\mathrm{C} 5$ & $0.62513(14)$ & $0.4679(4)$ & $0.9770(5)$ & $0.0568(8)$ \\
\hline H5A & 0.6336 & 0.5216 & 1.1185 & $0.068 *$ \\
\hline C6 & $0.58661(16)$ & $0.5931(5)$ & $0.8368(7)$ & $0.0829(12)$ \\
\hline H6A & 0.5476 & 0.6200 & 0.9094 & $0.124 *$ \\
\hline H6B & 0.5777 & 0.5412 & 0.6983 & $0.124^{*}$ \\
\hline $\mathrm{H} 6 \mathrm{C}$ & 0.6105 & 0.6967 & 0.8149 & $0.124 *$ \\
\hline $\mathrm{C} 7$ & $0.68777(14)$ & 0.4223 & $0.8702(5)$ & $0.0462(7)$ \\
\hline $\mathrm{C} 8$ & $0.74419(15)$ & $0.4799(4)$ & $0.9563(5)$ & $0.0577(8)$ \\
\hline H8A & 0.7436 & 0.5427 & 1.0852 & $0.069^{*}$ \\
\hline C9 & $0.80144(15)$ & $0.4470(4)$ & $0.8567(7)$ & $0.0697(10)$ \\
\hline H9A & 0.8391 & 0.4873 & 0.9163 & $0.084^{*}$ \\
\hline $\mathrm{C} 10$ & $0.80134(17)$ & $0.3551(5)$ & $0.6707(7)$ & $0.0689(10)$ \\
\hline C11 & $0.74733(18)$ & $0.2932(4)$ & $0.5777(5)$ & $0.0654(9)$ \\
\hline
\end{tabular}




$\begin{array}{lllll}\text { H11A } & 0.7489 & 0.2301 & 0.4491 & 0.078^{*} \\ \text { C12 } & 0.69042(15) & 0.3265(4) & 0.6789(5) & 0.0543(8) \\ \text { H12A } & 0.6532 & 0.2843 & 0.6184 & 0.065^{*}\end{array}$

Atomic displacement parameters $\left(\AA^{2}\right)$

\begin{tabular}{lllllll}
\hline & $U^{11}$ & $U^{22}$ & $U^{33}$ & $U^{12}$ & $U^{13}$ & $U^{23}$ \\
\hline S1 & $0.0499(6)$ & $0.0559(6)$ & $0.0379(5)$ & $-0.0056(6)$ & 0.000 & 0.000 \\
F1 & $0.0687(14)$ & $0.1167(19)$ & $0.164(2)$ & $-0.0047(14)$ & $0.0485(15)$ & $-0.030(2)$ \\
N1 & $0.0452(14)$ & $0.0502(15)$ & $0.0540(16)$ & $-0.0082(12)$ & $0.0032(11)$ & $-0.0028(11)$ \\
C2 & $0.0405(16)$ & $0.054(2)$ & $0.0489(18)$ & $-0.0028(15)$ & $-0.0043(14)$ & $-0.0073(16)$ \\
C3 & $0.0388(15)$ & $0.0544(19)$ & $0.0452(17)$ & $-0.0011(15)$ & $-0.0013(13)$ & $-0.0024(14)$ \\
C4 & $0.056(2)$ & $0.071(2)$ & $0.0396(15)$ & $-0.0115(15)$ & $-0.0020(13)$ & $-0.0040(14)$ \\
C5 & $0.0545(18)$ & $0.0513(19)$ & $0.0647(18)$ & $-0.0104(16)$ & $0.0112(15)$ & $-0.0111(16)$ \\
C6 & $0.065(2)$ & $0.060(2)$ & $0.123(3)$ & $0.0111(19)$ & $0.023(2)$ & $0.016(2)$ \\
C7 & $0.0468(17)$ & $0.0403(15)$ & $0.0514(17)$ & $-0.0063(14)$ & $0.0002(14)$ & $0.0001(13)$ \\
C8 & $0.0576(19)$ & $0.0492(17)$ & $0.0662(19)$ & $-0.0090(17)$ & $0.0002(16)$ & $-0.0104(18)$ \\
C9 & $0.047(2)$ & $0.064(2)$ & $0.099(3)$ & $-0.0114(17)$ & $-0.0023(19)$ & $-0.007(2)$ \\
C10 & $0.054(2)$ & $0.058(2)$ & $0.095(3)$ & $-0.0009(18)$ & $0.021(2)$ & $0.001(2)$ \\
C11 & $0.075(2)$ & $0.063(2)$ & $0.0577(19)$ & $0.000(2)$ & $0.011(2)$ & $-0.0053(18)$ \\
C12 & $0.0499(18)$ & $0.0573(19)$ & $0.0557(18)$ & $-0.0058(17)$ & $-0.0063(16)$ & $-0.0020(16)$ \\
& & & & & &
\end{tabular}

Geometric parameters $\left(\AA,{ }^{\circ}\right)$

\begin{tabular}{llll}
\hline $\mathrm{S} 1-\mathrm{C} 3^{\mathrm{i}}$ & $1.725(3)$ & $\mathrm{C} 6-\mathrm{H} 6 \mathrm{~A}$ & 0.9600 \\
$\mathrm{~S} 1-\mathrm{C} 3$ & $1.725(3)$ & $\mathrm{C} 6-\mathrm{H} 6 \mathrm{~B}$ & 0.9600 \\
$\mathrm{~F} 1-\mathrm{C} 10$ & $1.365(4)$ & $\mathrm{C} 6-\mathrm{H} 6 \mathrm{C}$ & 0.9600 \\
$\mathrm{~N} 1-\mathrm{C} 2$ & $1.264(4)$ & $\mathrm{C} 7-\mathrm{C} 8$ & $1.378(4)$ \\
$\mathrm{N} 1-\mathrm{C} 5$ & $1.473(4)$ & $\mathrm{C} 7-\mathrm{C} 12$ & $1.392(4)$ \\
$\mathrm{C} 2-\mathrm{C} 3$ & $1.450(4)$ & $\mathrm{C} 8-\mathrm{C} 9$ & $1.378(4)$ \\
$\mathrm{C} 2-\mathrm{H} 2 \mathrm{~A}$ & 0.9300 & $\mathrm{C} 8-\mathrm{H} 8 \mathrm{~A}$ & 0.9300 \\
$\mathrm{C} 3-\mathrm{C} 4$ & $1.360(4)$ & $\mathrm{C} 9-\mathrm{C} 10$ & $1.347(5)$ \\
$\mathrm{C} 4-\mathrm{C} 4$ & $\mathrm{C} 9-\mathrm{H} 9 \mathrm{~A}$ & 0.9300 \\
$\mathrm{C} 4-\mathrm{H} 4 \mathrm{~A}$ & $1.414(6)$ & $\mathrm{C} 10-\mathrm{C} 11$ & $1.363(5)$ \\
$\mathrm{C} 5-\mathrm{C} 7$ & 0.9300 & $\mathrm{C} 11-\mathrm{C} 12$ & $1.377(4)$ \\
$\mathrm{C} 5-\mathrm{C} 6$ & $1.518(4)$ & $\mathrm{C} 11-\mathrm{H} 11 \mathrm{~A}$ & 0.9300 \\
$\mathrm{C} 5-\mathrm{H} 5 \mathrm{~A}$ & $1.533(5)$ & $\mathrm{C} 12-\mathrm{H} 12 \mathrm{~A}$ & 0.9300 \\
& 0.9800 & $\mathrm{H} 6 \mathrm{~A}-\mathrm{C} 6-\mathrm{H} 6 \mathrm{C}$ & 109.5 \\
$\mathrm{C} 3-\mathrm{S} 1-\mathrm{C} 3$ & & $\mathrm{H} 6 \mathrm{~B}-\mathrm{C} 6-\mathrm{H} 6 \mathrm{C}$ & 109.5 \\
$\mathrm{C} 2-\mathrm{N} 1-\mathrm{C} 5$ & $91.4(2)$ & $\mathrm{C} 8-\mathrm{C} 7-\mathrm{C} 12$ & $117.6(3)$ \\
$\mathrm{N} 1-\mathrm{C} 2-\mathrm{C} 3$ & $116.8(3)$ & $\mathrm{C} 8-\mathrm{C} 7-\mathrm{C} 5$ & $120.8(3)$ \\
$\mathrm{N} 1-\mathrm{C} 2-\mathrm{H} 2 \mathrm{~A}$ & $123.2(3)$ & $\mathrm{C} 12-\mathrm{C} 7-\mathrm{C} 5$ & $121.6(3)$ \\
$\mathrm{C} 3-\mathrm{C} 2-\mathrm{H} 2 \mathrm{~A}$ & 118.4 & $\mathrm{C} 7-\mathrm{C} 8-\mathrm{C} 9$ & $121.9(3)$ \\
$\mathrm{C} 4-\mathrm{C} 3-\mathrm{C} 2$ & 118.4 & $\mathrm{C} 7-\mathrm{C} 8-\mathrm{H} 8 \mathrm{~A}$ & 119.1 \\
$\mathrm{C} 4-\mathrm{C} 3-\mathrm{S} 1$ & $127.5(3)$ & $\mathrm{C} 9-\mathrm{C} 8-\mathrm{H} 8 \mathrm{~A}$ & 119.1 \\
$\mathrm{C} 2-\mathrm{C} 3-\mathrm{S} 1$ & $111.5(2)$ & $\mathrm{C} 10-\mathrm{C} 9-\mathrm{C} 8$ & $118.2(3)$ \\
$\mathrm{C} 3-\mathrm{C} 4-\mathrm{C} 4 \mathrm{i}$ & $120.9(2)$ & $\mathrm{C} 10-\mathrm{C} 9-\mathrm{H} 9 \mathrm{~A}$ & 120.9 \\
$\mathrm{C} 3-\mathrm{C} 4-\mathrm{H} 4 \mathrm{~A}$ & $112.81(18)$ & &
\end{tabular}




$\begin{array}{llll}\mathrm{C} 4-\mathrm{C} 4-\mathrm{H} 4 \mathrm{~A} & 123.6 & \mathrm{C} 8-\mathrm{C} 9-\mathrm{H} 9 \mathrm{~A} & 120.9 \\ \mathrm{~N} 1-\mathrm{C} 5-\mathrm{C} 7 & 110.3(2) & \mathrm{C} 9-\mathrm{C} 10-\mathrm{C} 11 & 122.9(3) \\ \mathrm{N} 1-\mathrm{C} 5-\mathrm{C} 6 & 108.4(2) & \mathrm{C} 9-\mathrm{C} 10-\mathrm{F} 1 & 118.4(3) \\ \mathrm{C} 7-\mathrm{C} 5-\mathrm{C} 6 & 111.6(2) & \mathrm{C} 11-\mathrm{C} 10-\mathrm{F} 1 & 118.7(3) \\ \mathrm{N} 1-\mathrm{C} 5-\mathrm{H} 5 \mathrm{~A} & 108.8 & \mathrm{C} 10-\mathrm{C} 11-\mathrm{C} 12 & 118.4(3) \\ \mathrm{C} 7-\mathrm{C} 5-\mathrm{H} 5 \mathrm{~A} & 108.8 & \mathrm{C} 10-\mathrm{C} 11-\mathrm{H} 11 \mathrm{~A} & 120.8 \\ \mathrm{C} 6-\mathrm{C} 5-\mathrm{H} 5 \mathrm{~A} & 108.8 & \mathrm{C} 12-\mathrm{C} 11-\mathrm{H} 11 \mathrm{~A} & 120.8 \\ \mathrm{C} 5-\mathrm{C} 6-\mathrm{H} 6 \mathrm{~A} & 109.5 & \mathrm{C} 11-\mathrm{C} 12-\mathrm{C} 7 & 121.1(3) \\ \mathrm{C} 5-\mathrm{C} 6-\mathrm{H} 6 \mathrm{~B} & 109.5 & \mathrm{C} 11-\mathrm{C} 12-\mathrm{H} 12 \mathrm{~A} & 119.5 \\ \mathrm{H} 6 \mathrm{~A}-\mathrm{C} 6-\mathrm{H} 6 \mathrm{~B} & 109.5 & \mathrm{C} 7-\mathrm{C} 12-\mathrm{H} 12 \mathrm{~A} & \\ \mathrm{C} 5-\mathrm{C} 6-\mathrm{H} 6 \mathrm{C} & 109.5 & & -67.4(4) \\ & & \mathrm{C} 6-\mathrm{C} 5-\mathrm{C} 7-\mathrm{C} 12 & 1.0(5) \\ \mathrm{C} 5-\mathrm{N} 1-\mathrm{C} 2-\mathrm{C} 3 & -179.6(2) & \mathrm{C} 12-\mathrm{C} 7-\mathrm{C} 8-\mathrm{C} 9 & -176.9(3) \\ \mathrm{N} 1-\mathrm{C} 2-\mathrm{C} 3-\mathrm{C} 4 & 168.6(3) & \mathrm{C} 5-\mathrm{C} 7-\mathrm{C} 8-\mathrm{C} 9 & -0.4(5) \\ \mathrm{N} 1-\mathrm{C} 2-\mathrm{C} 3-\mathrm{S} 1 & -8.7(4) & \mathrm{C} 7-\mathrm{C} 8-\mathrm{C} 9-\mathrm{C} 10 & -0.2(5) \\ \mathrm{C} 3-\mathrm{S} 1-\mathrm{C} 3-\mathrm{C} 4 & -0.29(16) & \mathrm{C} 8-\mathrm{C} 9-\mathrm{C} 10-\mathrm{C} 11 & -179.1(3) \\ \mathrm{C} 3-\mathrm{S} 1-\mathrm{C} 3-\mathrm{C} 2 & 177.4(3) & \mathrm{C} 9-\mathrm{C} 10-\mathrm{C} 11-\mathrm{C} 12 & 0.0(6) \\ \mathrm{C} 2-\mathrm{C} 3-\mathrm{C} 4-\mathrm{C} 4 \mathrm{i} & -176.7(3) & \mathrm{F} 1-\mathrm{C} 10-\mathrm{C} 11-\mathrm{C} 12 & 179.0(3) \\ \mathrm{S} 1-\mathrm{C} 3-\mathrm{C} 4-\mathrm{C} 4 & \mathrm{C} 10-\mathrm{C} 11-\mathrm{C} 12-\mathrm{C} 7 & 0.6(5) \\ \mathrm{C} 2-\mathrm{N} 1-\mathrm{C} 5-\mathrm{C} 7 & 0.8(4) & \mathrm{C} 8-\mathrm{C} 7-\mathrm{C} 12-\mathrm{C} 11 & -1.1(4) \\ \mathrm{C} 2-\mathrm{N} 1-\mathrm{C} 5-\mathrm{C} 6 & 124.5(3) & \mathrm{C} 5-\mathrm{C} 7-\mathrm{C} 12-\mathrm{C} 11 & 176.7(3) \\ \mathrm{N} 1-\mathrm{C} 5-\mathrm{C} 7-\mathrm{C} 8 & -113.0(3) & -129.0(3) & \end{array}$

Symmetry code: (i) $-x+1,-y, z$.

(IV) 2,5-Bis $\{[(S)-(+)-1-(4-c h l o r o p h e n y l) e t h y l]$ iminomethyl\}thiophene

Crystal data

$\mathrm{C}_{22} \mathrm{H}_{20} \mathrm{Cl}_{2} \mathrm{~N}_{2} \mathrm{~S}$

$M_{r}=415.36$

Orthorhombic, $P 2{ }_{1} 2{ }_{1} 2$

$a=21.893(2) \AA$

$b=7.9212(6) \AA$

$c=6.2315(4) \AA$

$V=1080.66(15) \AA^{3}$

$Z=2$

$F(000)=432$

\section{Data collection}

Agilent Xcalibur (Atlas, Gemini) diffractometer

Radiation source: Enhance (Mo) X-ray Source Graphite monochromator

Detector resolution: 10.5564 pixels $\mathrm{mm}^{-1}$

$\omega$ scans

Absorption correction: multi-scan

CrysAlis PRO, (Agilent, 2013)

$T_{\min }=0.692, T_{\max }=1.000$
$D_{\mathrm{x}}=1.276 \mathrm{Mg} \mathrm{m}^{-3}$

Melting point: $434 \mathrm{~K}$

Mo $K \alpha$ radiation, $\lambda=0.71073 \AA$

Cell parameters from 2744 reflections

$\theta=3.7-21.5^{\circ}$

$\mu=0.41 \mathrm{~mm}^{-1}$

$T=298 \mathrm{~K}$

Prism, colorless

$0.52 \times 0.40 \times 0.07 \mathrm{~mm}$

14195 measured reflections

2743 independent reflections

1534 reflections with $I>2 \sigma(I)$

$R_{\text {int }}=0.058$

$\theta_{\max }=29.5^{\circ}, \theta_{\min }=3.3^{\circ}$

$h=-28 \rightarrow 27$

$k=-10 \rightarrow 9$

$l=-8 \rightarrow 8$ 


\section{Refinement}

Refinement on $F^{2}$

Least-squares matrix: full

$R\left[F^{2}>2 \sigma\left(F^{2}\right)\right]=0.052$

$w R\left(F^{2}\right)=0.117$

$S=1.01$

2743 reflections

124 parameters

0 restraints

Primary atom site location: structure-invariant direct methods

Secondary atom site location: difference Fourier map
Hydrogen site location: inferred from neighbouring sites

$\mathrm{H}$-atom parameters constrained

$w=1 /\left[\sigma^{2}\left(F_{\mathrm{o}}^{2}\right)+(0.0483 P)^{2}\right]$

where $P=\left(F_{\mathrm{o}}^{2}+2 F_{\mathrm{c}}{ }^{2}\right) / 3$

$(\Delta / \sigma)_{\max }<0.001$

$\Delta \rho_{\max }=0.13$ e $\AA^{-3}$

$\Delta \rho_{\text {min }}=-0.17$ e $\AA^{-3}$

Absolute structure: Flack $x$ determined using 465 quotients $\left[\left(I^{+}\right)-\left(I^{-}\right)\right] /\left[\left(I^{+}\right)+\left(I^{-}\right)\right]$(Parsons et al., 2013)

Absolute structure parameter: 0.10 (6)

Fractional atomic coordinates and isotropic or equivalent isotropic displacement parameters $\left(\AA^{2}\right)$

\begin{tabular}{lllll}
\hline & $x$ & $y$ & $z$ & $U_{\text {iso }} / U_{\text {eq }}$ \\
\hline S1 & 0.5000 & 1.0000 & $-0.13764(18)$ & $0.0590(4)$ \\
C11 & $0.15354(6)$ & $0.67176(16)$ & $0.4970(2)$ & $0.1059(5)$ \\
N1 & $0.41391(14)$ & $0.6958(3)$ & $-0.0839(5)$ & $0.0612(8)$ \\
C2 & $0.43185(16)$ & $0.7244(4)$ & $-0.2735(6)$ & $0.0589(10)$ \\
H2A & 0.4204 & 0.6496 & -0.3812 & $0.071^{*}$ \\
C3 & $0.46969(15)$ & $0.8689(4)$ & $-0.3311(5)$ & $0.0544(9)$ \\
C4 & $0.48309(16)$ & $0.9247(4)$ & $-0.5338(5)$ & $0.0640(10)$ \\
H4A & 0.4711 & 0.8688 & -0.6581 & $0.077^{*}$ \\
C5 & $0.37559(18)$ & $0.5438(4)$ & $-0.0520(7)$ & $0.0677(11)$ \\
H5A & 0.3625 & 0.5021 & -0.1927 & $0.081^{*}$ \\
C6 & $0.4148(2)$ & $0.4087(5)$ & $0.0553(9)$ & $0.0986(17)$ \\
H6A & 0.4484 & 0.3803 & -0.0369 & $0.148^{*}$ \\
H6B & 0.3905 & 0.3098 & 0.0810 & $0.148^{*}$ \\
H6C & 0.4302 & 0.4509 & 0.1892 & $0.148^{*}$ \\
C7 & $0.31964(17)$ & $0.5839(4)$ & $0.0796(5)$ & $0.0555(9)$ \\
C8 & $0.26313(19)$ & $0.5202(5)$ & $0.0255(7)$ & $0.0701(10)$ \\
H8A & 0.2593 & 0.4575 & -0.0999 & $0.084^{*}$ \\
C9 & $0.21198(19)$ & $0.5464(5)$ & $0.1512(7)$ & $0.0735(11)$ \\
H9A & 0.1744 & 0.5020 & 0.1109 & $0.088^{*}$ \\
C10 & $0.21746(18)$ & $0.6384(5)$ & $0.3351(6)$ & $0.0640(10)$ \\
C11 & $0.2728(2)$ & $0.7062(5)$ & $0.3945(6)$ & $0.0691(10)$ \\
H11A & 0.2761 & 0.7692 & 0.5199 & $0.083^{*}$ \\
C12 & $0.32346(18)$ & $0.6802(4)$ & $0.2665(5)$ & $0.0623(9)$ \\
H12A & 0.3607 & 0.7276 & 0.3056 & $0.075^{*}$ \\
& & & & \\
\hline
\end{tabular}

Atomic displacement parameters $\left(\AA^{2}\right)$

\begin{tabular}{lllllll}
\hline & $U^{11}$ & $U^{22}$ & $U^{33}$ & $U^{12}$ & $U^{13}$ & $U^{23}$ \\
\hline S1 & $0.0694(9)$ & $0.0596(7)$ & $0.0482(6)$ & $-0.0040(7)$ & 0.000 & 0.000 \\
C11 & $0.0878(9)$ & $0.1061(9)$ & $0.1238(10)$ & $0.0069(7)$ & $0.0384(8)$ & $0.0026(9)$ \\
N1 & $0.062(2)$ & $0.0569(18)$ & $0.0643(19)$ & $-0.0089(15)$ & $0.0064(15)$ & $0.0009(14)$ \\
C2 & $0.055(2)$ & $0.060(2)$ & $0.061(2)$ & $0.0015(17)$ & $-0.0031(19)$ & $-0.0056(18)$
\end{tabular}




\begin{tabular}{lllllll} 
C3 & $0.050(2)$ & $0.060(2)$ & $0.0523(19)$ & $0.0004(17)$ & $-0.0008(16)$ & $-0.0008(17)$ \\
C4 & $0.063(3)$ & $0.080(2)$ & $0.0487(18)$ & $-0.0130(18)$ & $-0.0011(17)$ & $-0.0059(17)$ \\
C5 & $0.071(3)$ & $0.057(2)$ & $0.075(2)$ & $-0.0098(18)$ & $0.015(2)$ & $-0.0050(18)$ \\
C6 & $0.085(3)$ & $0.064(2)$ & $0.147(4)$ & $0.011(2)$ & $0.040(3)$ & $0.019(3)$ \\
C7 & $0.061(2)$ & $0.0442(17)$ & $0.061(2)$ & $-0.0052(17)$ & $-0.0039(18)$ & $0.0020(16)$ \\
C8 & $0.073(3)$ & $0.061(2)$ & $0.076(2)$ & $-0.017(2)$ & $-0.002(2)$ & $-0.014(2)$ \\
C9 & $0.063(3)$ & $0.067(3)$ & $0.091(3)$ & $-0.0168(19)$ & $-0.003(2)$ & $-0.007(2)$ \\
C10 & $0.065(3)$ & $0.056(2)$ & $0.072(2)$ & $0.0010(19)$ & $0.007(2)$ & $0.007(2)$ \\
C11 & $0.081(3)$ & $0.068(2)$ & $0.059(2)$ & $-0.002(2)$ & $-0.003(2)$ & $-0.0067(18)$ \\
C12 & $0.059(2)$ & $0.065(2)$ & $0.063(2)$ & $-0.004(2)$ & $-0.010(2)$ & $-0.002(2)$ \\
\hline
\end{tabular}

Geometric parameters $\left(\AA,{ }^{o}\right)$

\begin{tabular}{|c|c|c|c|}
\hline $\mathrm{S} 1-\mathrm{C} 3$ & $1.724(3)$ & $\mathrm{C} 6-\mathrm{H} 6 \mathrm{~A}$ & 0.9600 \\
\hline $\mathrm{S} 1-\mathrm{C} 3^{\mathrm{i}}$ & $1.724(3)$ & $\mathrm{C} 6-\mathrm{H} 6 \mathrm{~B}$ & 0.9600 \\
\hline $\mathrm{C} 11-\mathrm{C} 10$ & $1.746(4)$ & $\mathrm{C} 6-\mathrm{H} 6 \mathrm{C}$ & 0.9600 \\
\hline $\mathrm{N} 1-\mathrm{C} 2$ & $1.265(4)$ & $\mathrm{C} 7-\mathrm{C} 8$ & $1.378(5)$ \\
\hline $\mathrm{N} 1-\mathrm{C} 5$ & $1.481(4)$ & $\mathrm{C} 7-\mathrm{C} 12$ & $1.394(4)$ \\
\hline $\mathrm{C} 2-\mathrm{C} 3$ & $1.458(5)$ & $\mathrm{C} 8-\mathrm{C} 9$ & $1.382(5)$ \\
\hline $\mathrm{C} 2-\mathrm{H} 2 \mathrm{~A}$ & 0.9300 & $\mathrm{C} 8-\mathrm{H} 8 \mathrm{~A}$ & 0.9300 \\
\hline $\mathrm{C} 3-\mathrm{C} 4$ & $1.370(4)$ & $\mathrm{C} 9-\mathrm{C} 10$ & $1.363(5)$ \\
\hline $\mathrm{C} 4-\mathrm{C} 4^{\mathrm{i}}$ & $1.404(7)$ & C9-H9A & 0.9300 \\
\hline $\mathrm{C} 4-\mathrm{H} 4 \mathrm{~A}$ & 0.9300 & $\mathrm{C} 10-\mathrm{C} 11$ & $1.376(5)$ \\
\hline $\mathrm{C} 5-\mathrm{C} 7$ & $1.508(5)$ & $\mathrm{C} 11-\mathrm{C} 12$ & $1.382(5)$ \\
\hline $\mathrm{C} 5-\mathrm{C} 6$ & $1.527(5)$ & $\mathrm{C} 11-\mathrm{H} 11 \mathrm{~A}$ & 0.9300 \\
\hline $\mathrm{C} 5-\mathrm{H} 5 \mathrm{~A}$ & 0.9800 & $\mathrm{C} 12-\mathrm{H} 12 \mathrm{~A}$ & 0.9300 \\
\hline $\mathrm{C} 3-\mathrm{S} 1-\mathrm{C}^{\mathrm{i}}$ & $91.3(2)$ & $\mathrm{H} 6 \mathrm{~A}-\mathrm{C} 6-\mathrm{H} 6 \mathrm{C}$ & 109.5 \\
\hline $\mathrm{C} 2-\mathrm{N} 1-\mathrm{C} 5$ & $116.6(3)$ & $\mathrm{H} 6 \mathrm{~B}-\mathrm{C} 6-\mathrm{H} 6 \mathrm{C}$ & 109.5 \\
\hline $\mathrm{N} 1-\mathrm{C} 2-\mathrm{C} 3$ & $123.2(3)$ & $\mathrm{C} 8-\mathrm{C} 7-\mathrm{C} 12$ & $117.3(4)$ \\
\hline $\mathrm{N} 1-\mathrm{C} 2-\mathrm{H} 2 \mathrm{~A}$ & 118.4 & $\mathrm{C} 8-\mathrm{C} 7-\mathrm{C} 5$ & $121.3(3)$ \\
\hline $\mathrm{C} 3-\mathrm{C} 2-\mathrm{H} 2 \mathrm{~A}$ & 118.4 & $\mathrm{C} 12-\mathrm{C} 7-\mathrm{C} 5$ & $121.4(3)$ \\
\hline $\mathrm{C} 4-\mathrm{C} 3-\mathrm{C} 2$ & $127.0(3)$ & $\mathrm{C} 7-\mathrm{C} 8-\mathrm{C} 9$ & $122.2(4)$ \\
\hline $\mathrm{C} 4-\mathrm{C} 3-\mathrm{S} 1$ & $111.6(3)$ & $\mathrm{C} 7-\mathrm{C} 8-\mathrm{H} 8 \mathrm{~A}$ & 118.9 \\
\hline $\mathrm{C} 2-\mathrm{C} 3-\mathrm{S} 1$ & $121.3(2)$ & $\mathrm{C} 9-\mathrm{C} 8-\mathrm{H} 8 \mathrm{~A}$ & 118.9 \\
\hline $\mathrm{C} 3-\mathrm{C} 4-\mathrm{C} 4^{\mathrm{i}}$ & $112.8(2)$ & $\mathrm{C} 10-\mathrm{C} 9-\mathrm{C} 8$ & $119.0(4)$ \\
\hline $\mathrm{C} 3-\mathrm{C} 4-\mathrm{H} 4 \mathrm{~A}$ & 123.6 & $\mathrm{C} 10-\mathrm{C} 9-\mathrm{H} 9 \mathrm{~A}$ & 120.5 \\
\hline $\mathrm{C} 4-\mathrm{C} 4-\mathrm{H} 4 \mathrm{~A}$ & 123.6 & $\mathrm{C} 8-\mathrm{C} 9-\mathrm{H} 9 \mathrm{~A}$ & 120.5 \\
\hline $\mathrm{N} 1-\mathrm{C} 5-\mathrm{C} 7$ & $111.2(3)$ & $\mathrm{C} 9-\mathrm{C} 10-\mathrm{C} 11$ & $120.8(4)$ \\
\hline $\mathrm{N} 1-\mathrm{C} 5-\mathrm{C} 6$ & $108.1(3)$ & $\mathrm{C} 9-\mathrm{C} 10-\mathrm{Cl1}$ & $119.8(3)$ \\
\hline $\mathrm{C} 7-\mathrm{C} 5-\mathrm{C} 6$ & $111.5(3)$ & $\mathrm{C} 11-\mathrm{C} 10-\mathrm{Cl} 1$ & $119.4(3)$ \\
\hline $\mathrm{N} 1-\mathrm{C} 5-\mathrm{H} 5 \mathrm{~A}$ & 108.7 & $\mathrm{C} 10-\mathrm{C} 11-\mathrm{C} 12$ & $119.5(3)$ \\
\hline $\mathrm{C} 7-\mathrm{C} 5-\mathrm{H} 5 \mathrm{~A}$ & 108.7 & $\mathrm{C} 10-\mathrm{C} 11-\mathrm{H} 11 \mathrm{~A}$ & 120.2 \\
\hline $\mathrm{C} 6-\mathrm{C} 5-\mathrm{H} 5 \mathrm{~A}$ & 108.7 & $\mathrm{C} 12-\mathrm{C} 11-\mathrm{H} 11 \mathrm{~A}$ & 120.2 \\
\hline $\mathrm{C} 5-\mathrm{C} 6-\mathrm{H} 6 \mathrm{~A}$ & 109.5 & $\mathrm{C} 11-\mathrm{C} 12-\mathrm{C} 7$ & $121.0(4)$ \\
\hline $\mathrm{C} 5-\mathrm{C} 6-\mathrm{H} 6 \mathrm{~B}$ & 109.5 & $\mathrm{C} 11-\mathrm{C} 12-\mathrm{H} 12 \mathrm{~A}$ & 119.5 \\
\hline $\mathrm{H} 6 \mathrm{~A}-\mathrm{C} 6-\mathrm{H} 6 \mathrm{~B}$ & 109.5 & $\mathrm{C} 7-\mathrm{C} 12-\mathrm{H} 12 \mathrm{~A}$ & 119.5 \\
\hline $\mathrm{C} 5-\mathrm{C} 6-\mathrm{H} 6 \mathrm{C}$ & 109.5 & & \\
\hline
\end{tabular}




\begin{tabular}{|c|c|c|c|}
\hline $\mathrm{C} 5-\mathrm{N} 1-\mathrm{C} 2-\mathrm{C} 3$ & $179.8(3)$ & $\mathrm{C} 6-\mathrm{C} 5-\mathrm{C} 7-\mathrm{C} 12$ & $74.9(4)$ \\
\hline $\mathrm{N} 1-\mathrm{C} 2-\mathrm{C} 3-\mathrm{C} 4$ & $-168.8(4)$ & $\mathrm{C} 12-\mathrm{C} 7-\mathrm{C} 8-\mathrm{C} 9$ & $-1.2(5)$ \\
\hline $\mathrm{N} 1-\mathrm{C} 2-\mathrm{C} 3-\mathrm{S} 1$ & $7.3(5)$ & $\mathrm{C} 5-\mathrm{C} 7-\mathrm{C} 8-\mathrm{C} 9$ & $175.8(3)$ \\
\hline 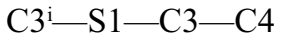 & $0.42(19)$ & $\mathrm{C} 7-\mathrm{C} 8-\mathrm{C} 9-\mathrm{C} 10$ & $0.0(6)$ \\
\hline $\mathrm{C} 3{ }^{\mathrm{i}}-\mathrm{S} 1-\mathrm{C} 3-\mathrm{C} 2$ & $-176.3(4)$ & $\mathrm{C} 8-\mathrm{C} 9-\mathrm{C} 10-\mathrm{C} 11$ & $0.8(6)$ \\
\hline $\mathrm{C} 2-\mathrm{C} 3-\mathrm{C} 4-\mathrm{C} 4^{\mathrm{i}}$ & $175.3(4)$ & $\mathrm{C} 8-\mathrm{C} 9-\mathrm{C} 10-\mathrm{Cl} 1$ & $-179.6(3)$ \\
\hline $\mathrm{S} 1-\mathrm{C} 3-\mathrm{C} 4-\mathrm{C} 4^{\mathrm{i}}$ & $-1.1(5)$ & $\mathrm{C} 9-\mathrm{C} 10-\mathrm{C} 11-\mathrm{C} 12$ & $-0.3(6)$ \\
\hline $\mathrm{C} 2-\mathrm{N} 1-\mathrm{C} 5-\mathrm{C} 7$ & $-132.2(3)$ & $\mathrm{C} 11-\mathrm{C} 10-\mathrm{C} 11-\mathrm{C} 12$ & $-179.9(3)$ \\
\hline $\mathrm{C} 2-\mathrm{N} 1-\mathrm{C} 5-\mathrm{C} 6$ & $105.1(4)$ & $\mathrm{C} 10-\mathrm{C} 11-\mathrm{C} 12-\mathrm{C} 7$ & $-1.0(6)$ \\
\hline $\mathrm{N} 1-\mathrm{C} 5-\mathrm{C} 7-\mathrm{C} 8$ & $137.3(4)$ & $\mathrm{C} 8-\mathrm{C} 7-\mathrm{C} 12-\mathrm{C} 11$ & $1.7(5)$ \\
\hline $\mathrm{C} 6-\mathrm{C} 5-\mathrm{C} 7-\mathrm{C} 8$ & $-102.0(4)$ & $\mathrm{C} 5-\mathrm{C} 7-\mathrm{C} 12-\mathrm{C} 11$ & $-175.3(3)$ \\
\hline $\mathrm{N} 1-\mathrm{C} 5-\mathrm{C} 7-\mathrm{C} 12$ & $-45.8(4)$ & & \\
\hline
\end{tabular}

Symmetry code: (i) $-x+1,-y+2, z$. 